\title{
Determinantes del embarazo en adolescentes y su impacto en los ejes fundamentales de ingreso y nivel educativo
}

\section{Determinants of adolescent pregnancy and their impact on the fundamental axes of income and educational level}

Yenizer Alexandra Tafur-Bonilla ${ }^{1}$, Olga Lucía Barbosa-Campos², Edwin Leonardo Méndez-Ortiz ${ }^{3} \bowtie$

\author{
${ }^{1}$ Estudiante de pregrado de Administración Financiera. Semillero, \\ Universidad Minuto de Dios, Bogotá, Colombia. \\ 2 Estudiante de pregrado de Administración Financiera. Semillero, \\ Universidad Minuto de Dios, Bogotá, Colombia. \\ ${ }^{3}$ Candidato a Doctor en Administración, investigador, Universidad \\ Minuto de Dios, Bogotá, Colombia.
}

Universidad Minuto de Dios, Bogotá, Colombia. Correo electrónico: emendez@uniminuto.edu

Recibido: 22 de agosto del 2017 Aprobado: 15 de diciembre del 2017 Disponible en línea: 1 de enero del 2018

Cómo citar este artículo: Tafur-Bonilla, Y. A., Barbosa-Campos, O. L. y Méndez- Ortiz, E. L. (2018). Determinantes del embarazo en adolescentes y su impacto en los ejes fundamentales de ingreso y nivel educativo, Cooperativismo \& Desarrollo, 113(26). doi: https://doi.org/10.16925/co.v26i113.2191

\section{Resumen}

Introducción: en este artículo se busca medir el impacto socioeconómico de un embarazo en mujeres adolescentes en aspectos clave como, por ejemplo, el ingreso y el nivel educativo.

Metodología: se realizó una encuesta a las estudiantes de la Universidad Minuto de Dios de la modalidad Virtual y a Distancia, para la cual se tomó como muestra 201 encuestas, con el fin de determinar los modelos propuestos tanto en los determinantes del embarazo en adolescentes como en su impacto en el nivel educativo y en el ingreso actual. Las mujeres encuestadas se dividieron en tres grupos, distribuidos de la siguiente manera: madres en su adolescencia con pareja en la actualidad, madres en su adolescencia solteras en la actualidad y mujeres que en su adolescencia no hayan sido madres.

Resultados: después de realizar un análisis a regresiones lineales propuestas, se encontraron diferentes ejes: el grupo familiar, el estado civil de las mujeres y el ingreso a la vida laboral con relación al embarazo en la adolescencia.

Conclusiones: se encontró que los ejes estudiados generan un impacto en la adolescencia y en la vida actual de cualquier mujer desde su adolescencia hasta la actualidad.

Palabras clave: embarazo adolescente, impactos, ingresos, nivel educativo, variable endógena. 


\title{
Determinants of adolescent pregnancy and their impact on the fundamental axes of income and educational level
}

\author{
Abstract \\ Introduction: The present article aims at measuring socioeconomic impact of women's adolescent \\ pregnancy in key aspects such as income and educational level. \\ Methodology: A survey to Minuto de Dios University students of the Virtual and Distance modality was \\ conducted. The sample comprised 201 surveys and sought to prove the proposed models for determi- \\ ning adolescent pregnancy and also its impacts on educational level and current income. The surveyed \\ women were divided into three categories: adolescent mothers with a current couple, currently single \\ adolescent mothers and women who did not become mothers during adolescence.
}

Results: After analyzing the proposed linear regressions, different axes were found: family group, women's marital status and their beginning to work in relation to adolescent pregnancy.

Conclusions: It was found that the axes studied impact in adolescence and in the current life of any woman from her adolescence to the present.

Keywords: adolescent pregnancy, impacts, income, educational level, endogenous variable.

\section{Determinantes da gravidez em adolescentes e seu impacto em eixos fundamentais como a renda e a escolaridade}

\begin{abstract}
Resumo
Introdução: este artigo busca medir o impacto socioeconômico da gravidez em mulheres adolescentes em aspectos chave como, por exemplo, a renda e a escolaridade.

Metodologia: realizou-se uma sondagem com as alunas da Universidade Minuto de Dios da modalidade Virtual e a Distância. A amostra foram 201 sondagens, e 0 objetivo foi determinar os modelos propostos em relação aos determinantes da gravidez na adolescência e ao impacto na escolaridade e a renda atual. As mulheres inquiridas foram divididas em três grupos, assim distribuídos: mães na adolescência com parceiro no momento, mães na adolescência solteiras no momento e mulheres que não foram mães na adolescência.

Resultados: após a realização de uma análise das regressões lineares propostas, encontraram-se diferentes eixos: 0 grupo familiar, o estado civil das mulheres e 0 ingresso na vida profissional em relação à gravidez na adolescência.

Conclusões: encontrou-se que os eixos estudados geram impacto na adolescência e na vida atual de qualquer mulher desde a adolescência até o presente.
\end{abstract}

Palavras-chave: gravidez na adolescência, impactos, renda, escolaridade, variável endógena. 


\section{Introducción}

Bogotá es una de las ciudades en las que se presenta un mayor índice de embarazos de adolescentes, y en donde - según estudios que se publicaron a principios del 2015- el 14\% de los jóvenes de edades entre los 14 y los 19 años ya tienen su primer hijo. En este sentido, ¿qué impactos en el nivel de ingresos y en el nivel educativo conlleva este fenómeno?

Con el paso del tiempo se ha hecho énfasis en las consecuencias psicológicas y sociales de esta anomalía. Sin embargo, la primera base de la economía de un país son las familias, de manera que cabe preguntarse: ¿Cuáles son los efectos colaterales que tiene el hecho de que una adolescente en una edad de difícil enlistamiento laboral sea madre? Esto, dado que en la mayoría de los casos presentan una educación media terminada o inconclusa, lo que las deja en una situación en la que apremian las necesidades de un niño, por lo que están sujetas a lo que el mercado les ofrezca.

El desenlace de las diferentes situaciones que una joven madre debe tratar de aventajar genera un efecto dominó en la economía colombiana, ya que a una joven con un nivel de vida media se le presentan los suficientes obstáculos tanto educativos como laborales para subsistir, y a esto se le suma la responsabilidad de un menor.

De acuerdo con lo reseñado, en el proyecto se buscó medir el impacto económico de un grupo objetivo de 201 personas que vive esta situación desde la perspectiva de tres diferentes situaciones: mujeres que no tienen hijos en su adolescencia, mujeres que tienen hijos en su adolescencia y son madres cabeza de hogar, y mujeres que tienen hijos en su adolescencia y cuentan con una pareja. Con el fin de analizar los cambios en calidad de vida que se han generado en el trascurso de su rol según sea la situación se han estudiado 67 personas por cada subgrupo de investigación, así como de comparar las condiciones y características de cada uno a partir del análisis de las diferencias, los cambios y las desventajas de cada subgrupo objetivo en el embarazo adolescente, y así demostrar los cambios e impactos que tiene esta situación.

\section{Fundamento teórico}

El embarazo en adolescentes "constituye un evento vital de importantes repercusiones en distintas dimensiones de la mujer: estudio, estructura familiar, ocupación, proyecto de vida y estado civil" (Salazar, Rodríguez y Daza, 2007.) Con el transcurso del tiempo ha sido una situación que se incrementa día a día, pues el hecho de que no solo afecte el ámbito social de una población específica, sino que también genere efectos en los aspectos económicos, familiares y personales, puede generar cambios en la calidad de vida tanto de la adolescente como de la familia. Siempre se ha comprendido que la edad y el comportamiento de un(a) adolescente hacen parte de una etapa de la vida en la que muchas veces se acaban de formalizar muchas ideas, de esclarecer otras y, en algunas ocasiones, la rebeldía es uno de los comportamientos más tradicionales para el rango de estas edades. Si este es el caso, las ideas equivocadas y la falta de conocimientos son causa de que se tomen decisiones erróneas o no planeadas - como es el caso de un embarazo a temprana edad, en la cual en muchas ocasiones no se es consecuente con las ideas y los actos propios-, de manera que no existe responsabilidad propiamente sobre lo que se decide y la familia directamente se ve afectada, no solo por el crecimiento de esta, sino porque la economía del hogar debe cambiar de forma radical.

Según el Departamento Administrativo de Estadística (DANE) y el Ministerio de Educación de Colombia, la calidad de vida de las mujeres - específicamente en edades adolescentes- se encuentra inserta en las condiciones personales y en el entorno social en el que se desarrolle. En estas edades es muy inestable la toma de decisiones, de lo que son un claro ejemplo los indicadores de deserción estudiantil, los cuales demuestran que, por problemas externos a los jóvenes, bien sean económicos o bien familiares, ellos deben abandonar sus estudios, a lo que se suman situaciones como, por ejemplo, un embarazo a temprana edad, por el que se presentan factores que afectan la economía familiar, los proyectos personales $y$, por ende, el inicio de una vida laboral a una temprana edad. El problema radica en los impactos que implica traer un ser humano a tan temprana edad, cuando aún no se es responsable de su propia vida, de sus gastos o no se tienen propósitos claros, pues no se han terminado en muchas ocasiones los estudios necesarios que ayudan a encontrar un trabajo que solvente todas las necesidades. Además, la carga adicional que se le adhiere a la familia de la adolescente al acoger a una persona más que se debe mantener y darle un mejor futuro. 
Cambios como estos propician que esta problemática se perciba no solo en términos del aumento de la población de manera rápida y esporádica, sino también en relación con el costo y los posibles cambios económicos de la vida de una adolescente al quedar en embarazo.

A lo largo de la historia de la humanidad, sin importar la religión o la nacionalidad, hemos buscado explicar los diferentes aspectos que rodean las conductas del ser humano y las implicaciones de sus actos. Esto nos lleva a tratar de comprender el creciente índice de embarazos en adolescentes, una temática que se ha tratado de explicar desde el comportamiento psicológico y social por diferentes entidades y organizaciones a nivel mundial, así como enfocar investigaciones a sus causas, mas no a sus consecuencias. No obstante, en pocas ocasiones se aborda la temática desde el punto de vista económico a partir del nivel educativo, ámbito desde el cual queremos comprender y tratar dicha problemática, de manera que a continuación se detallan conceptos y teorías involucradas con dichos ámbitos.

Según la oms, en el contexto económico y social, las adolescentes con embarazos precoces tienden a afectar estos ejes fundamentales, ya que se ven obligadas a dejar la escuela para generar ingresos que solventen las necesidades de este nuevo integrante de la familia. Generar más ingresos por necesidad, así como un embarazo a tan temprana edad, "puede bloquear las posibilidades de continuar una transición hacia la vida adulta acorde con los planes de vida que quisieran para sí mismas" (Mendoza y Subiría, 2013, p. 31).

Además de generar repercusiones en sus propias vidas, según el director del Fondo Poblacional de Naciones Unidas para América Latina y el Caribe, Luis Mora, este fenómeno afecta el desarrollo de Latinoamérica, pues cuando las adolescentes se ven con la urgencia de generar ingresos para sostener a su bebé, se ven sometidas a aceptar empleos informales, lo que genera más pobreza y un efecto en las economías de sus países. De acuerdo con las investigaciones realizadas por el Banco Mundial, las personas con bajas condiciones socioeconómicas, al quedar en embarazo, disminuyen el aprovechamiento de oportunidades que podrían mejorar sus condiciones actuales.

Asimismo, otro factor que puede sosegar la economía en Latinoamérica es la rapidez con la que aumenta la población adolescente en embarazo, ya que "el fenómeno se evidencia en una mayor pobreza, deserción escolar en edades tempranas y violencia sexual, entre otros" (Colprensa, 2015). La misma conclusión es planteada por Mejía-Mendoza et al. (2015), lo que evidencia cómo el impacto que tiene el embarazo en las adolescentes implica directamente su educación y, por ende, sus ingresos, y de esta manera el tipo de educación que pueden recibir los hijos de estas jóvenes madres.

Sin embargo, las adolescentes no son las únicas involucradas en un embarazo a temprana edad, ya que dentro de la concepción de un ser humano se encuentra involucrado un hombre, sin importar el escenario. No obstante, las consecuencias se ven en un mayor porcentaje en las mujeres que en los hombres, lo que abre una brecha en los ingresos de la madre del doble con relación a la que abre para el padre. El estado civil de las adolescentes tiene un alto índice de cambio desde el momento en el que estas quedan en embarazo: cuando nace su primer hijo esto tiene consecuencias económicas según sea el caso (Salazar-Arango, Acosta-Murcia, LozanoRestrepo y Quintero-Camacho, 2008).

A su vez, las mujeres que quedan en embarazo a temprana edad, según Welti (2000), presentan mayor porcentaje de divorcios, pues este autor asume que no tienen un grupo familiar estable o presentan inestabilidad en su vida en pareja. Si bien es cierto que el embarazo es un cambio fuerte para la vida de cualquier persona (sin distingo de edad), para una adolescente es más difícil continuar su vida en aspectos académicos, laborales y familiares, pues existe la preocupación mayor de ser responsable por un hijo y sus necesidades.

Las mujeres comienzan a temprana edad su vida laboral cuando, por las necesidades que se deben cubrir y porque en ocasiones sus familias dejan de apoyarlas (Parada, 2005), los ingresos no son suficientes para cubrir los gastos que representa un bebé y algunas mujeres buscan conseguir una pareja que les ayude a cubrir estos gastos pero, en caso de no conseguirlo, estas mujeres toman cualquier oportunidad laboral que se les presente (incluso si su remuneración no es suficiente), con el fin de generar la estabilidad para su hijo y para ellas.

\section{Metodología}

La metodología aplicada en este proceso tuvo un enfoque cuantitativo, para lo cual se realizó una encuesta diseñada con el fin de valorar los ejes fundamentales de estudio para cada uno de los grupos 
objetivo con base en un modelo econométrico (Logit binomial), el cual permite observar los resultados de impactos relacionados con las variables de la investigación.

\section{Caracterización de la población encuestada}

Con el fin de definir el rango específico de madre adolescente se usa el concepto, tomado de la Organización Panamericana de la Salud (ops), por el cual se considera y contextualiza a una madre adolescente como aquella mujer que tuvo su primer hijo antes de los 20 años.

Así, se obtiene la siguiente clasificación en tres grupos: Madres en su adolescencia con pareja en la actualidad, Madres en su adolescencia solteras en la actualidad, y Mujeres que en su adolescencia no hayan sido madres.

\section{Grupo 1: Madres en su adolescencia con pareja en la actualidad}

De acuerdo con la aplicación del instrumento de investigación, los resultados recopilados en la encuesta y en la estadística descriptiva de esta para este grupo son los que se presentan a continuación.

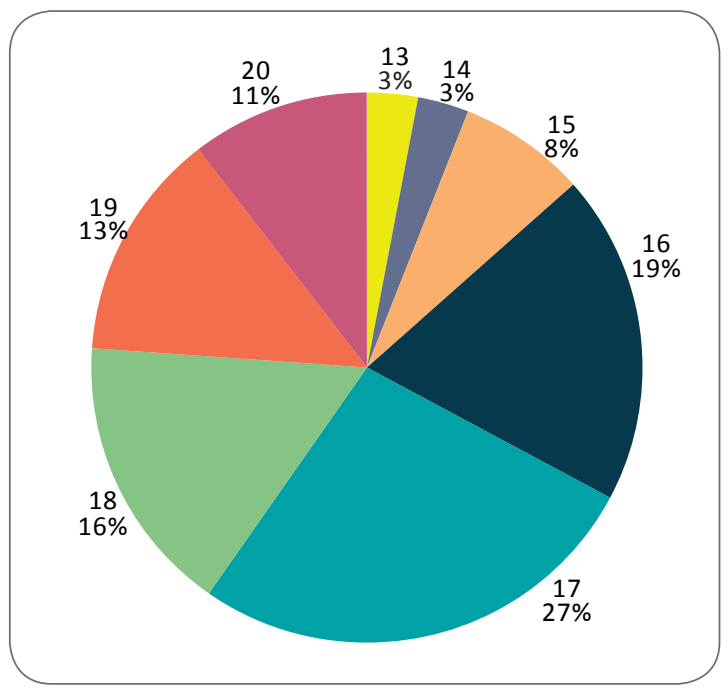

Figura 1. Edad en la que quedó en embarazo. Elaboración propia.

Del 100\% de las encuestadas que pertenecían a este grupo, se encontró que el $27 \%$ tuvo su primer embarazo a los 17 años, el 19\% a los 16, el 16\% a los
18 , el $13 \%$ a los 19 , el $11 \%$ a los 20 , el $8 \%$ a los 15 , el $3 \%$ a los 14 , y el $3 \%$ restante a los 13 años.

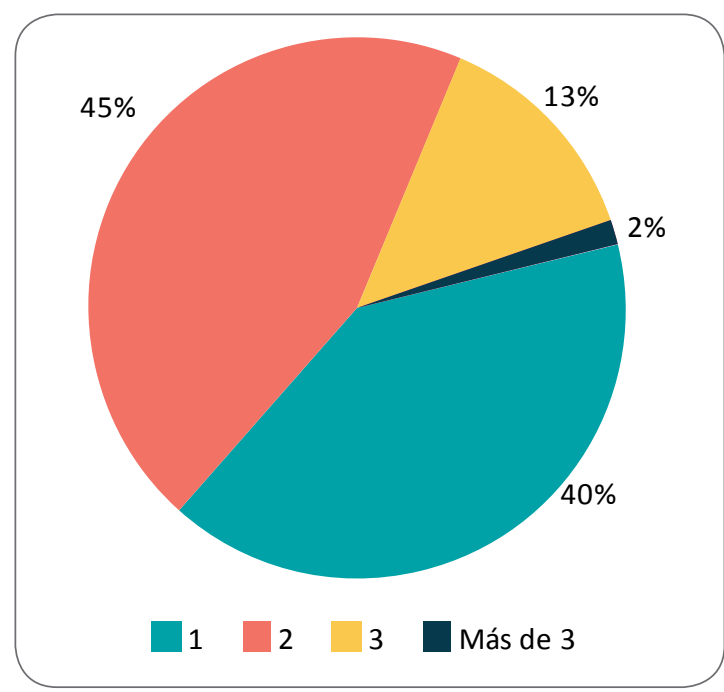

Figura 2. Cantidad de hijos actualmente. Elaboración propia.

Con respecto al número de hijos que tiene este grupo de mujeres en la actualidad, el $45 \%$ tiene dos, el $40 \%$ tiene un hijo, mientras que el $13 \%$ tiene tres, y el $2 \%$ restante tiene más de tres hijos.

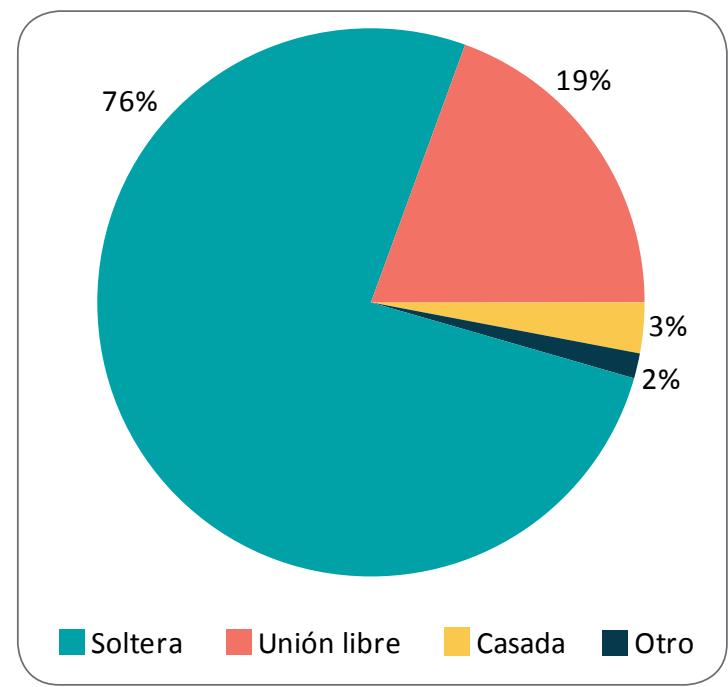

Figura 3. Estado civil en el momento de quedar embarazada. Elaboración propia.

En el momento de quedar en embarazo, el $76 \%$ de las mujeres eran solteras, el 19\% vivía en unión libre, el $3 \%$ se encontraba casada y el $2 \%$ en 
otro estado civil diferente a los nombrados, para lo cual se asume que quizás se encontraban en un noviazgo.

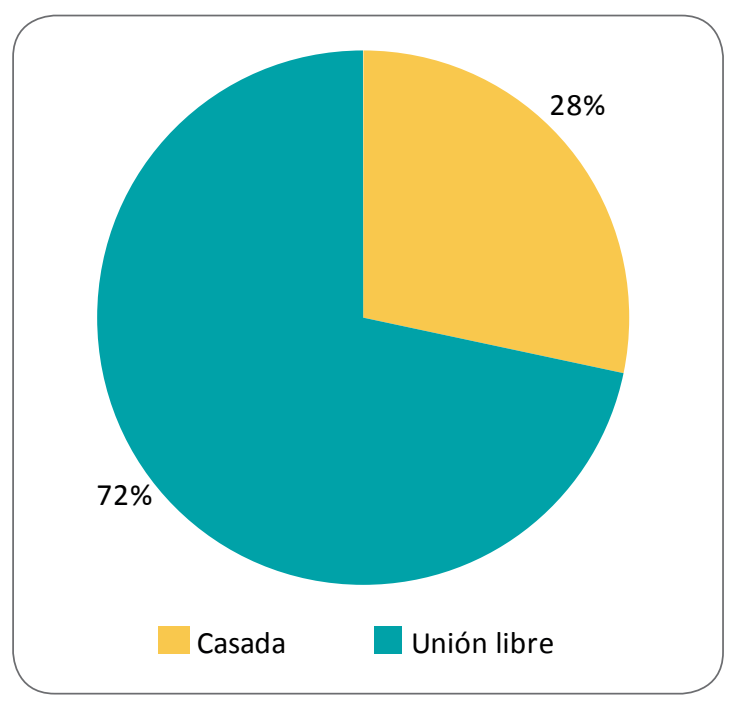

Figura 4. Estado civil actual. Elaboración propia.

Con respecto al estado civil de este grupo de la población, el $72 \%$ vive en unión libre, y el $28 \%$ restante se encuentra casado.

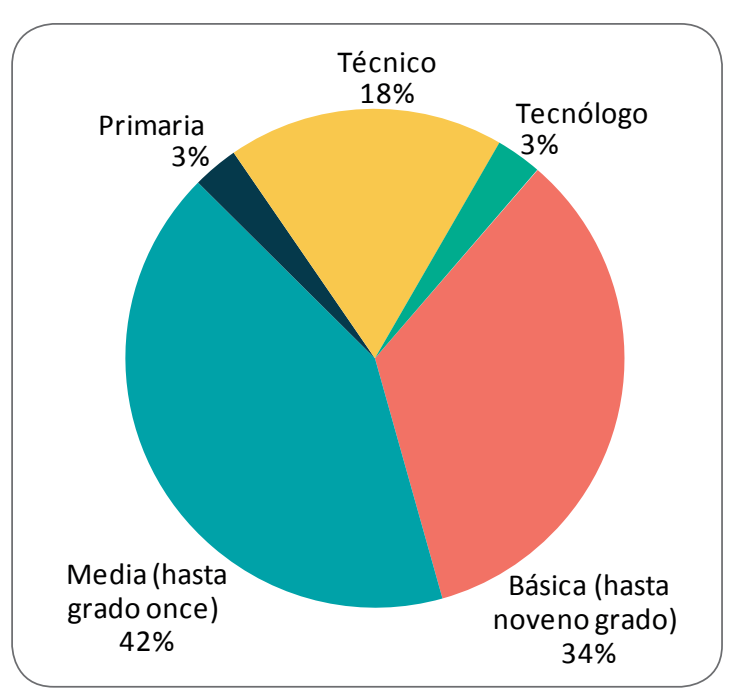

Figura 5. Nivel educativo en su primer embarazo. Elaboración propia.

El 42\% había culminado una educación media hasta el grado 11, el 34\% una educación media hasta grado noveno, el $18 \%$ un nivel técnico, el $3 \%$ un nivel tecnológico y el $3 \%$ restante solo la primaria.

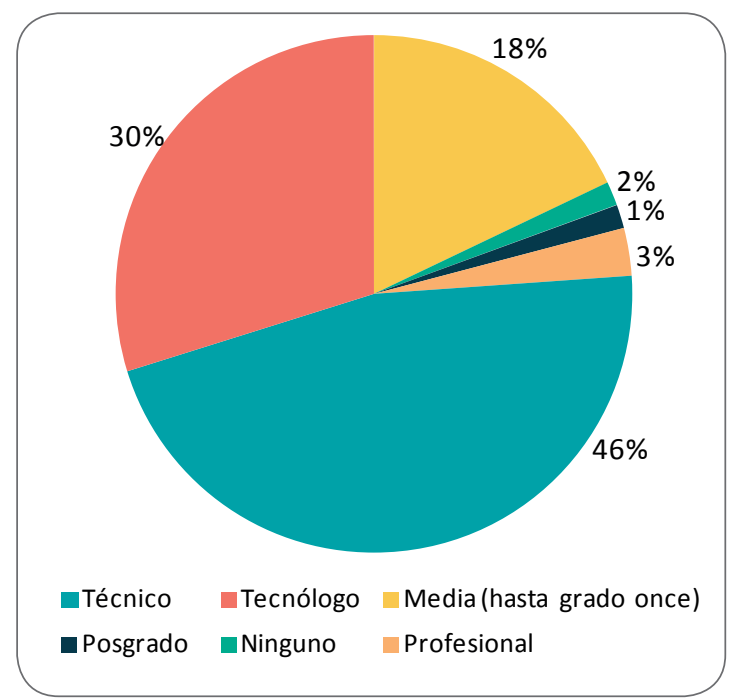

Figura 6. Nivel educativo actual. Elaboración propia.

En la actualidad, el $46 \%$ de estas mujeres han alcanzado un nivel técnico, el 30\% uno de tecnólogo, el $18 \%$ hasta grado once, el $3 \%$ culminó un nivel profesional, el $2 \%$ no tiene ninguno nivel educativo culminado en su totalidad y el $1 \%$ cuenta con un posgrado.

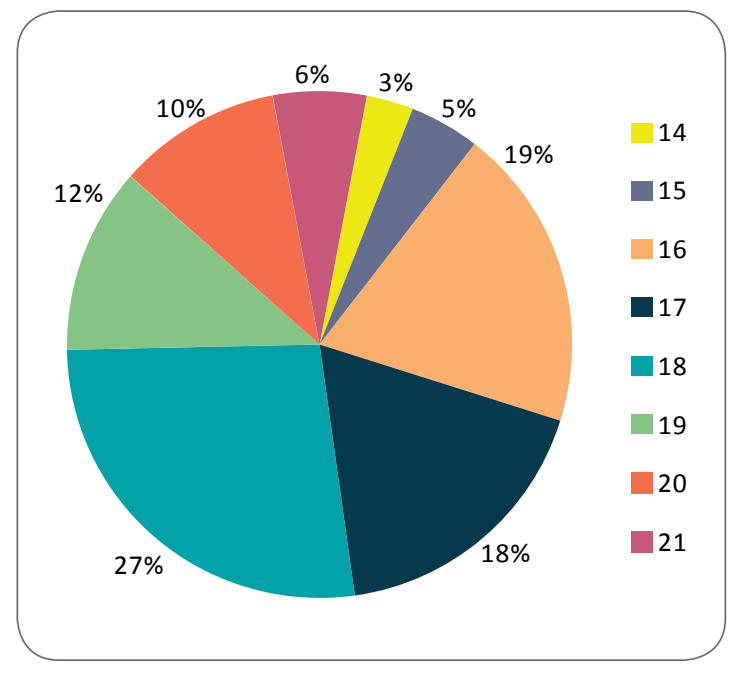

Figura 7. ¿A qué edad inició su vida laboral? Elaboración propia.

El 27\% de las mujeres que quedaron en embarazo antes de los 20 años comenzó su vida laboral entre los 13 y los 16 años, el 18\% a los 17, el 27\% a los 18 , y el $28 \%$ restante ingresó a la vida laboral entre los 19 y los 21 años. 


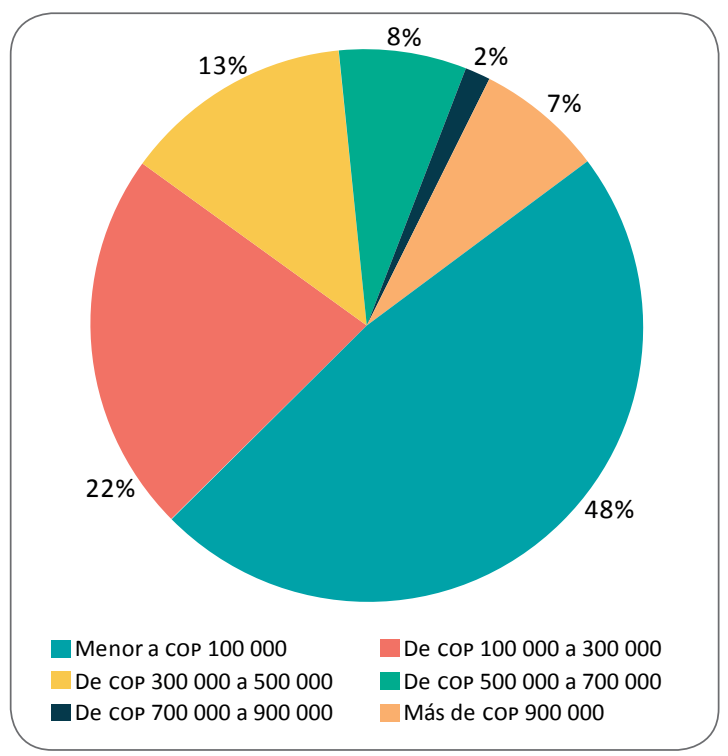

Figura 8. Ingresos promedio al momento de quedar en embarazo por primera vez. Elaboración propia.

Con respecto a los ingresos en el momento del embarazo, el 22\% de las mujeres tenían ingresos entre los COP 100000 y los COP 300 000, el 13\% entre los COP 300000 y COP 500 000, el 8\% tenía ingresos entre los COP 500000 y los COP 700 000, un $2 \%$ tenía ingresos entre los COP 700000 y los COP 900000 , el 7\% de más de cop 900000 , y el $48 \%$ restante de mujeres encuestadas tenían ingresos menores a COP 100000.

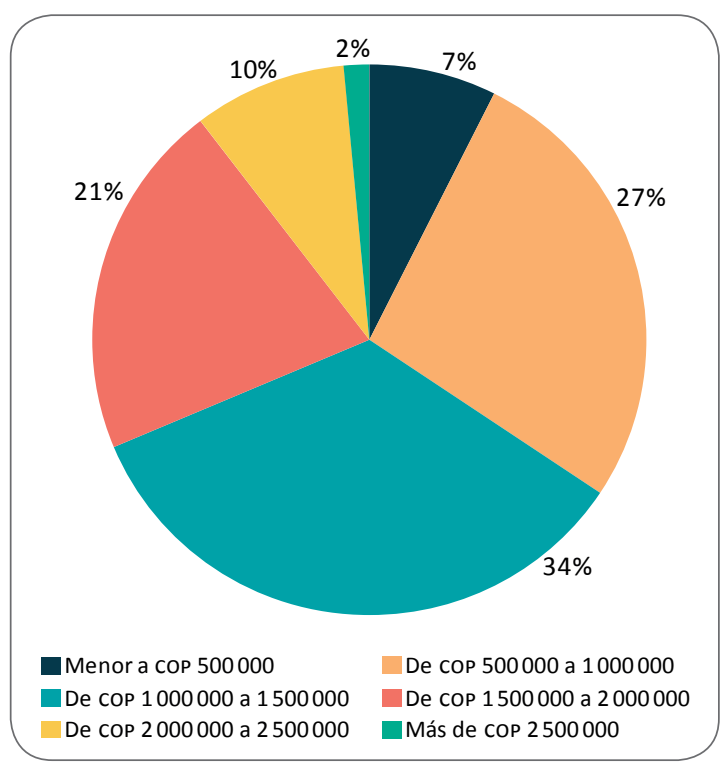

Figura 9. Ingresos promedio en la actualidad. Elaboración propia.
En la actualidad, el $10 \%$ de las mujeres presenta un ingreso menor a COP 500000 , el $28 \%$ entre COP 500000 y COP 1000000 , el 31\% de entre COP 1000000 y cOP 1500 000, el 17\% tiene un ingreso actual entre COP 1500000 y COP 2000 000, el $12 \%$ tiene ingresos de entre COP 2000000 y COP 2500000 ; el restante $2 \%$ de las mujeres encuestadas tienen ingresos mayores de COP 2500000 .

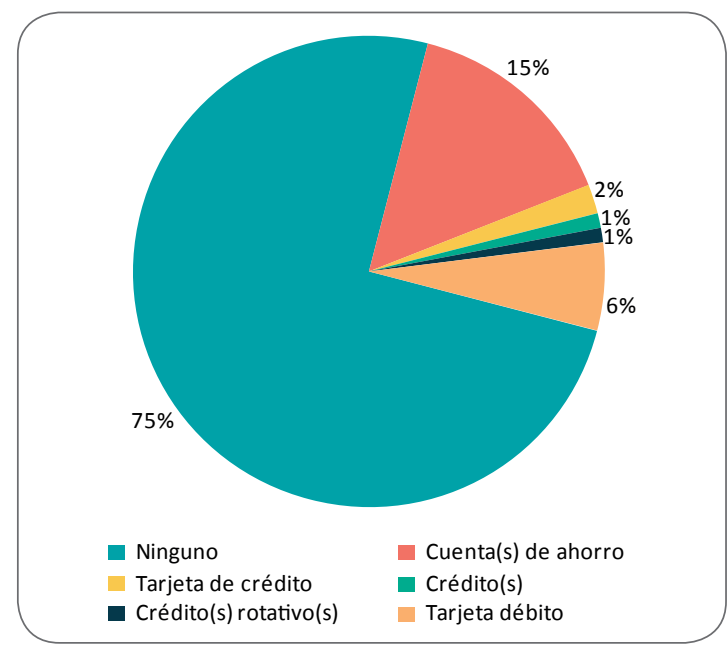

Figura 10. Productos del sistema financiero en el momento de quedar en embarazo por primera vez. Elaboración propia.

El 15\% contaba con una cuenta de ahorros, el $2 \%$ tenía una tarjeta de crédito, el $1 \%$ tenía un crédito, el $1 \%$ un crédito rotativo, y el $6 \%$ contaba con una tarjeta débito, mientras que el $75 \%$ no contaba con ninguno de los productos financieros nombrados.

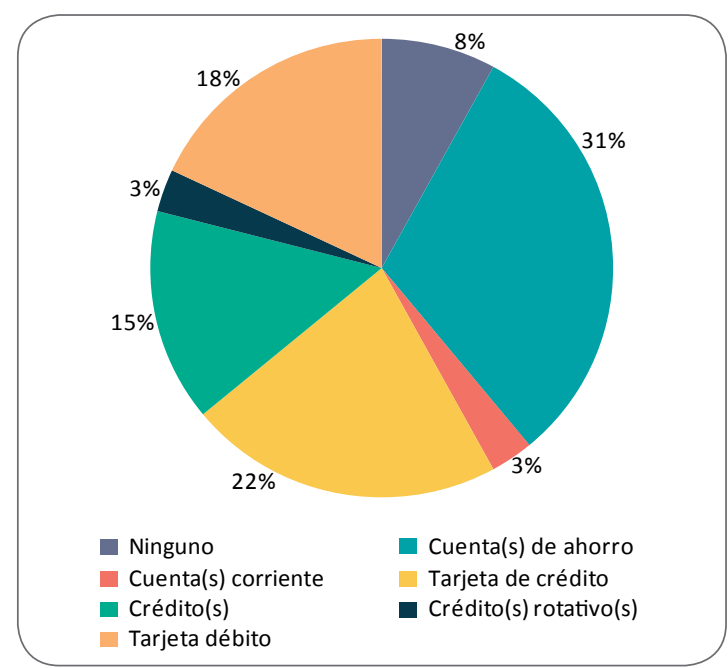

Figura 11. Productos del sistema financiero con los que actualmente cuenta. Elaboración propia. 
El $31 \%$ cuenta con una cuenta de ahorros, el $22 \%$ tiene mínimo una tarjeta de crédito, el $18 \%$ tiene una tarjeta débito, el 15\% tiene un crédito, el 8\% no maneja ningún producto financiero, el 3\% tiene una cuenta corriente y el 3\% restante tiene un crédito rotativo.

\section{Grupo 2: Madres en su adolescencia y cabeza de hogar en la actualidad}

Los resultados de la encuesta realizada para este segundo grupo y su estadística descriptiva se presentan a continuación.

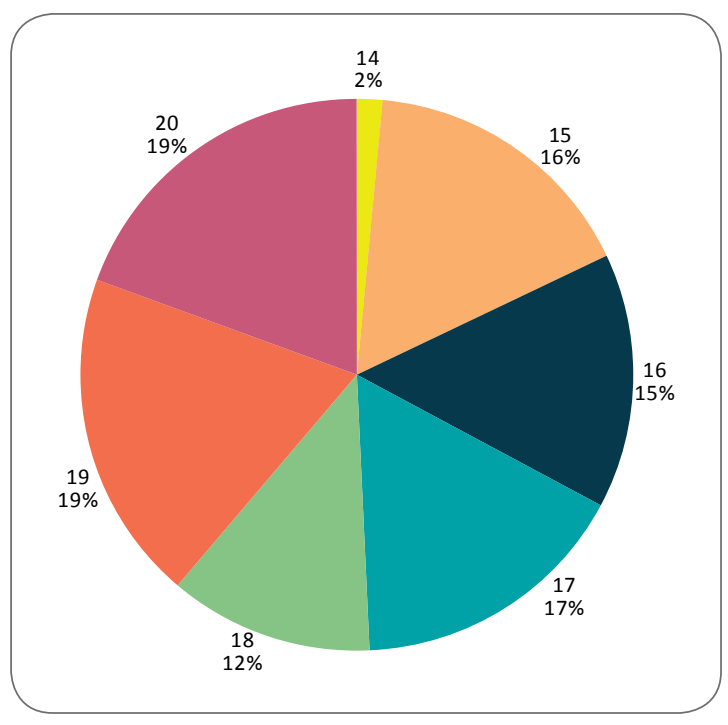

Figura 12. ¿Qué edad tenía cuando tuvo su primer embarazo? Elaboración propia.

El 19\% tuvo su primer hijo a los 20 años, el 19\% a los 19 , el $12 \%$ a los 18 , el $12 \%$ a los 17 , el $17 \%$ a los 17 también, el $15 \%$ a los 15 , el $16 \%$ a los 15 y el $2 \%$ restante a los 14 años.

El 77\% de las mujeres tiene un solo hijo, el 21\% tiene dos, y el $2 \%$ restante tres.

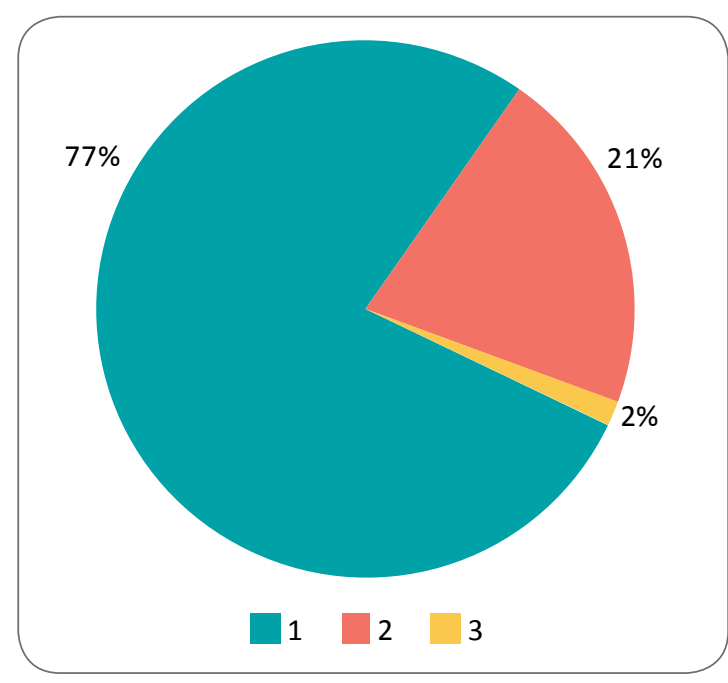

Figura 13. Número de hijos. Elaboración propia.

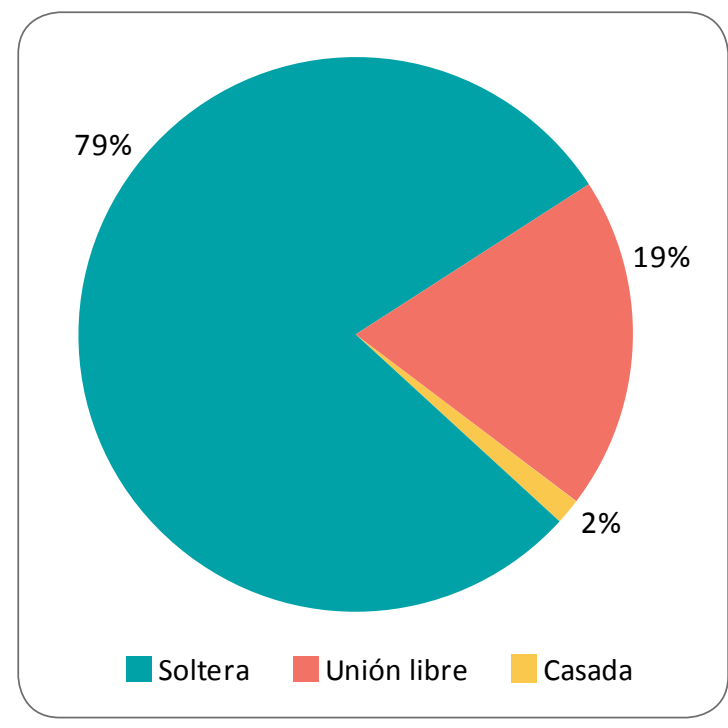

Figura 14. Estado civil en el momento de quedar en embarazo. Elaboración propia.

En el momento de quedar en embarazo, el 79\% de las mujeres eran solteras, el 19\% vivía en unión libre y el $2 \%$ estaban casadas. 


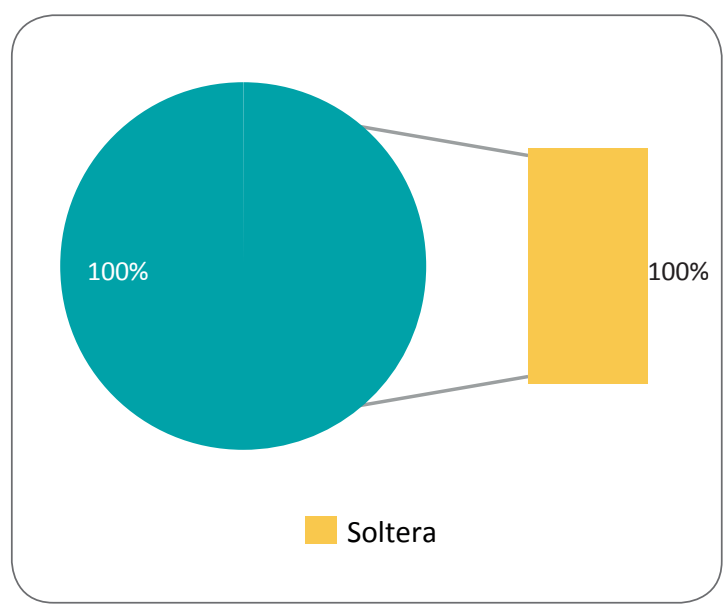

Figura 15. Estado civil actual. Elaboración propia.

En la actualidad, el 100\% de las mujeres de este grupo son madres solteras.

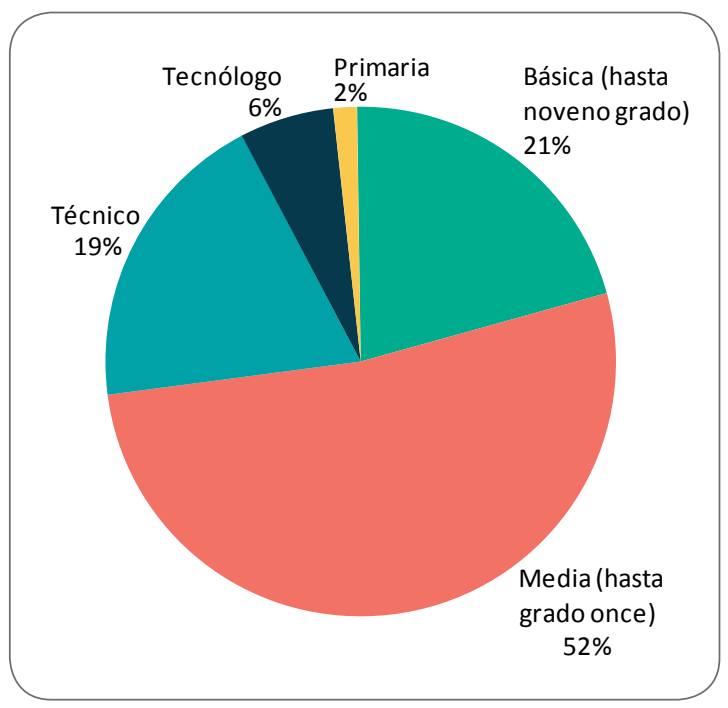

Figura 16. Nivel educativo que culminó en el momento de quedar embarazada. Elaboración propia.

El nivel educativo con el que contaban las mujeres en el momento de quedar en embarazo, es: el 52\% hasta grado 11 , el $21 \%$ hasta grado noveno, el 19\% tenía un nivel técnico de educación, el 6\% alcanzó a culminar un nivel tecnólogo y el $2 \%$ restante la primaria.

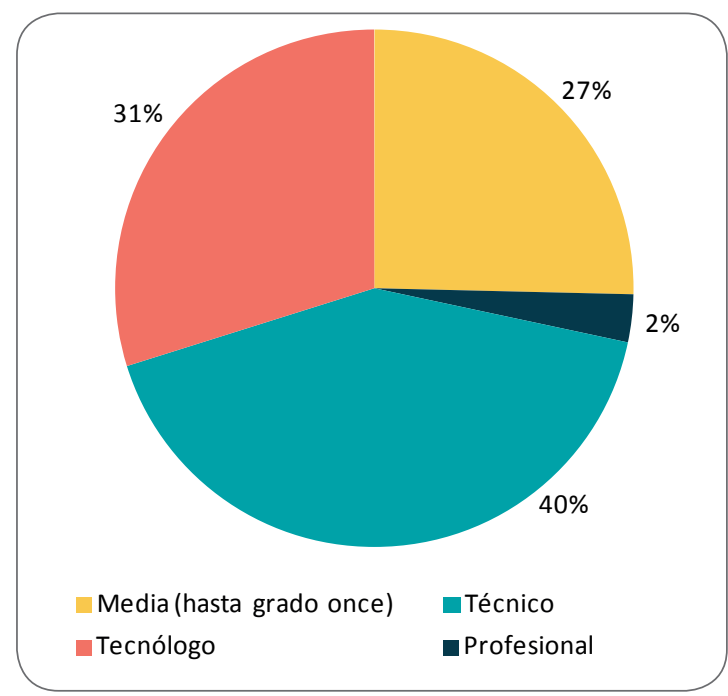

Figura 17. Nivel educativo actual. Elaboración propia.

Hoy en día, el $40 \%$ tiene un nivel técnico culminado, el 31\% un nivel tecnólogo, el 27\% cursó hasta grado 11 y el $2 \%$ completó en el nivel profesional una carrera.

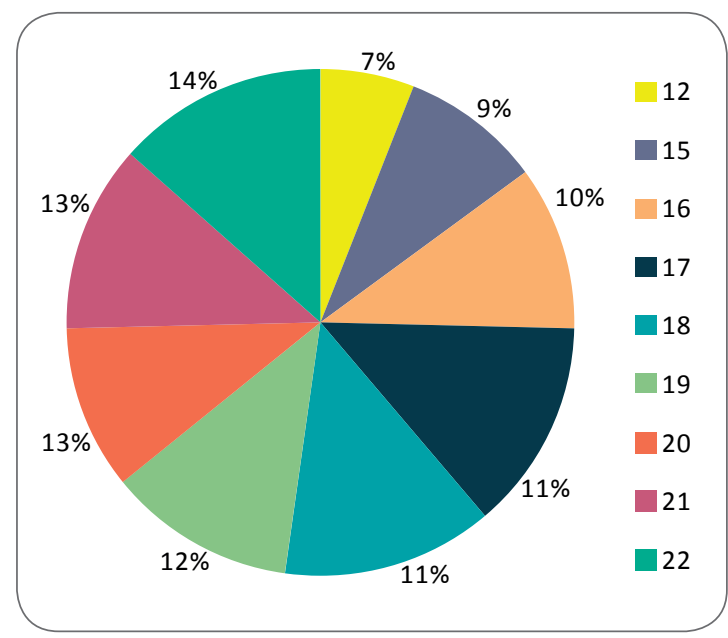

Figura 18. ¿A qué edad inició su vida laboral? Elaboración propia.

De este grupo de mujeres, el 7\% inició su vida laboral a los 12 años, el 9\% a los 15, el 10\% lo inició a los 16, el 22\% inició entre los 17 y los 18 años, el $25 \%$ entre los 19 y los 20 años, y el 27\% restante inició su vida laboral entre los 21 y los 22 años. 


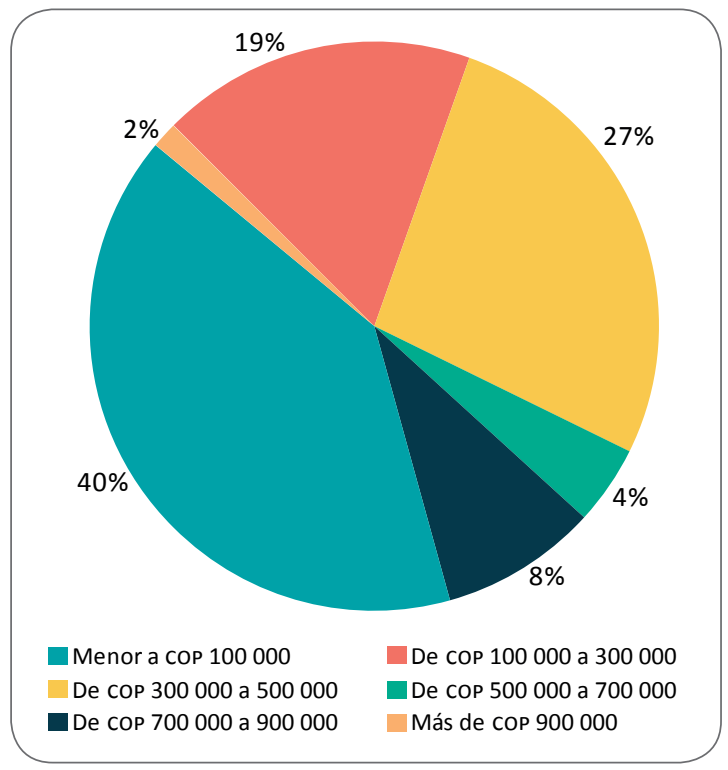

Figura 19. Ingreso promedio en el momento de quedar en embarazo por primera vez. Elaboración propia.

Con relación a los ingresos que se tenían en el momento de quedar en embarazo, el $40 \%$ presentaba ingresos menores a cop 100000 , el $27 \%$ entre cop 300000 y cop 500 000, y el 19\% entre los COP 100000 y COP 300000 , el 14\% restante presentaba ingresos entre COP 500000 y mayores a COP 900000 .

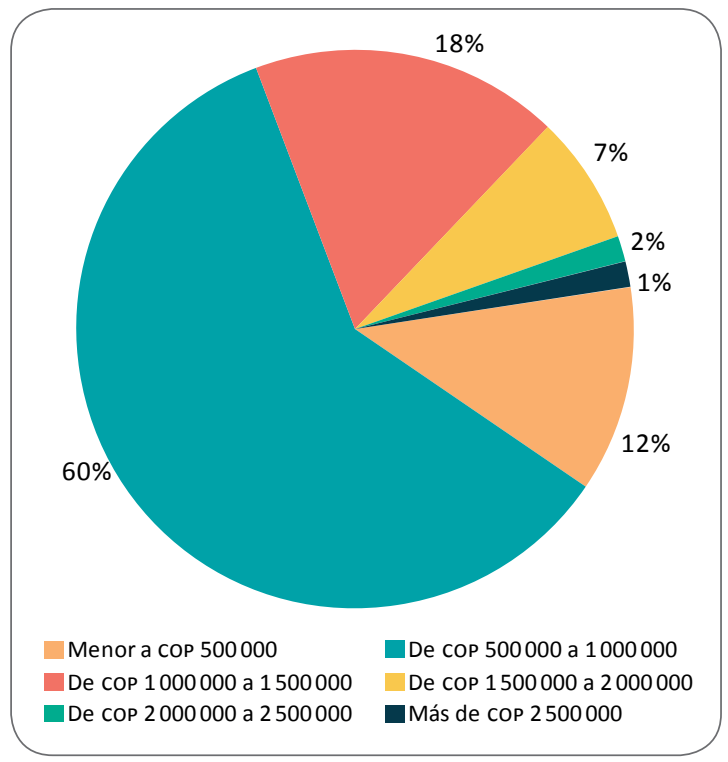

Figura 20. Ingreso promedio en la actualidad. Elaboración propia.
En la actualidad, solo el $8 \%$ tiene ingresos menores a COP 500 000, el 54\% entre COP 500000 y COP 1000 000, el 31\% entre COP 1000000 y cOP 2000000 , y el 7\% restante tiene ingresos mayores a COP 2000000 .

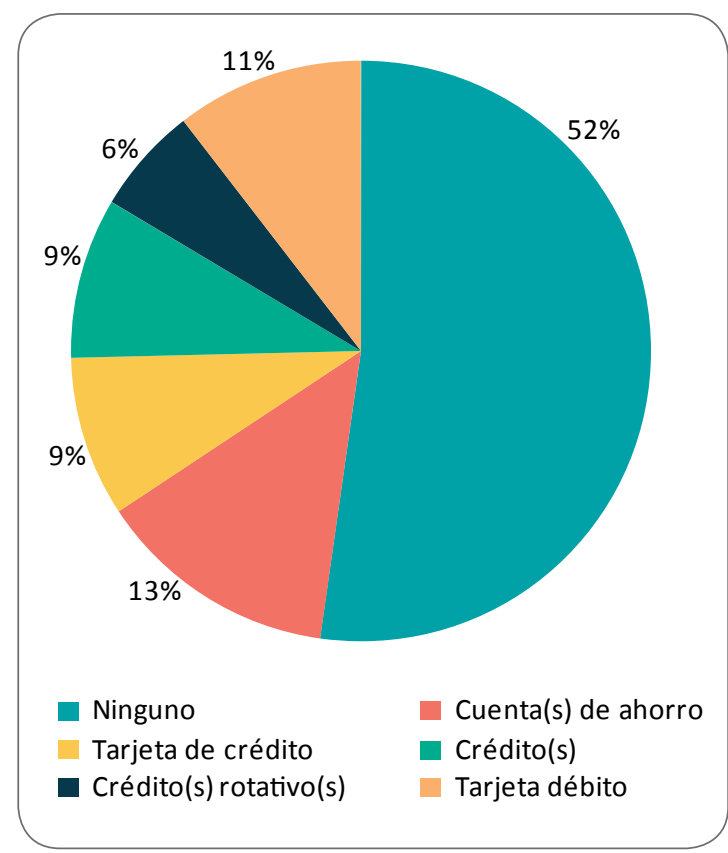

Figura 21. Productos del sistema financiero en el momento de quedar en embarazo por primera vez. Elaboración propia.

En el momento de quedar en embarazo, el 52\% no contaba con ningún producto financiero, el 13\% contaba con una cuenta de ahorros, el $11 \%$ con una tarjeta débito, el $9 \%$ con una tarjeta de crédito, el $9 \%$ con créditos en el momento de quedar en embarazo y el $6 \%$ restante con un crédito rotativo.

En la actualidad, el $31 \%$ de las mujeres cuenta con una cuenta de ahorros, el 5\% con una cuenta corriente, el $22 \%$ con una tarjeta de crédito, el $12 \%$ con un crédito, el $12 \%$ con una tarjeta débito, el $8 \%$ cuenta con un crédito rotativo, y solo el $10 \%$ no cuenta con ninguno de los productos financieros nombrados. 


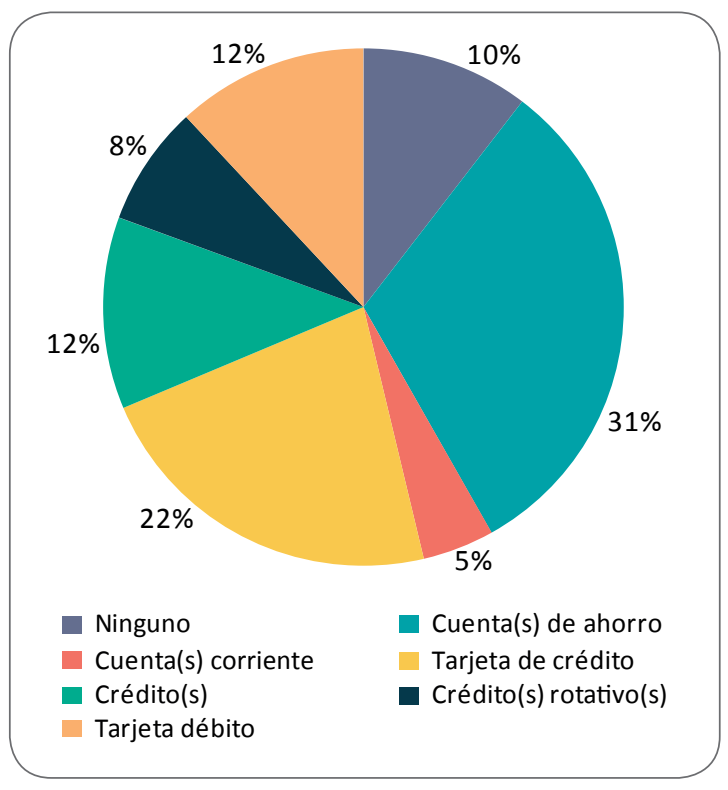

Figura 22. Productos del sistema financiero con los que cuenta en la actualidad. Elaboración propia.

\section{Grupo 3: Mujeres que en su adolescencia no hayan sido madres}

Los resultados para este grupo de la investigación frente a la encuesta realizada y su estadística descriptiva se presentan a continuación.

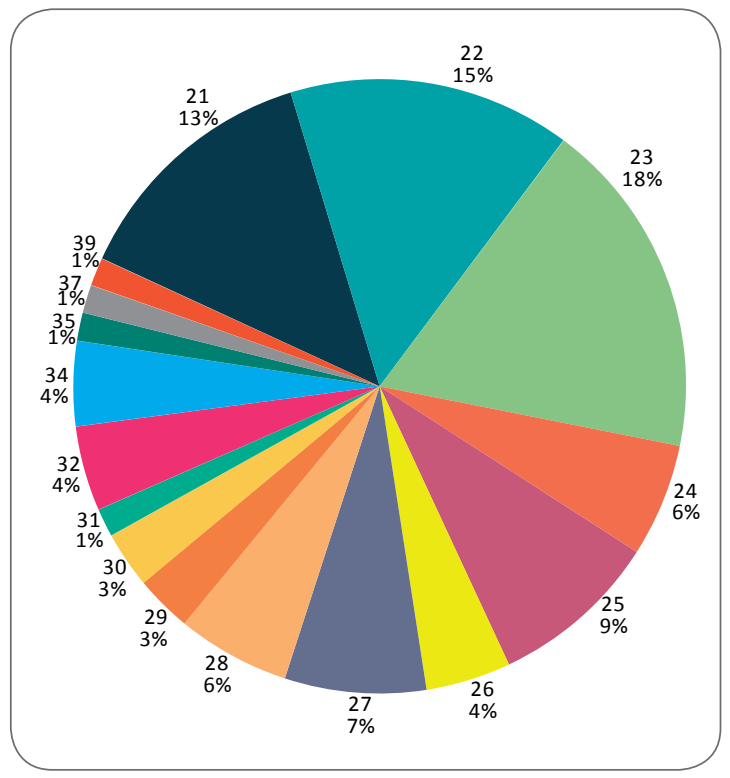

Figura 23. Edad actual. Elaboración propia.
Con respecto a la edad actual de las mujeres que completaron la encuesta, el $61 \%$ se encuentra en el rango de los 21 a los 25 años, el 23\% se encuentra entre los 25 y los 30 años de edad, y el 16\% entre los 31 y los 34 años.

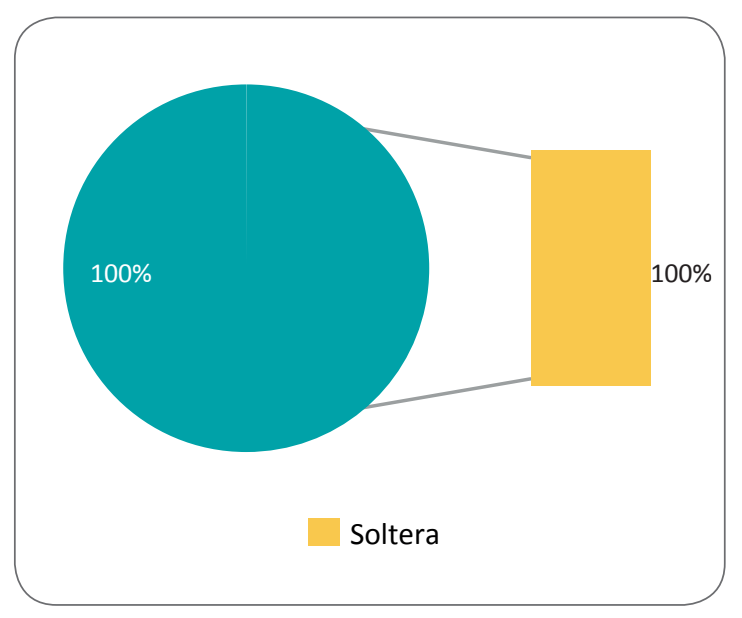

Figura 24. Estado civil antes de los 20 años. Elaboración propia.

Antes de cumplir 20 años, en este grupo de mujeres el $100 \%$ eran solteras, es decir, no tenían un compromiso oficial, ni un esposo.

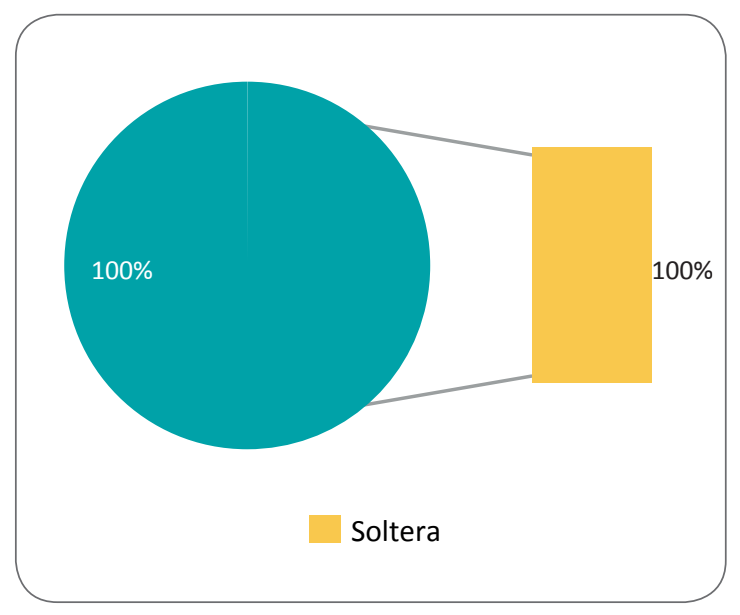

Figura 25. Estado civil actual. Elaboración propia. 
Con relación al estado civil actual de este grupo de mujeres, el $100 \%$ son solteras.

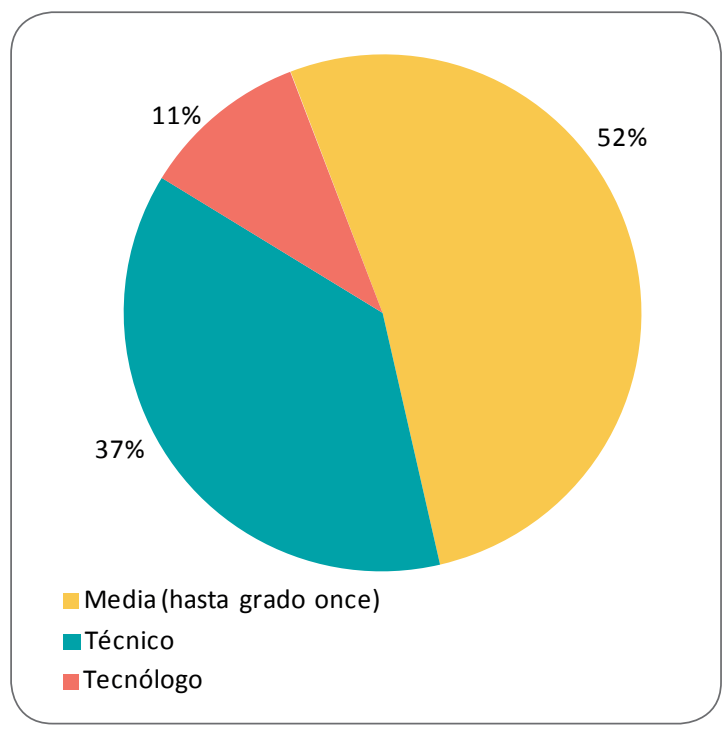

Figura 26. Nivel educativo antes de cumplir 20 años. Elaboración propia.

Con relación al nivel educativo que tenían antes de cumplir los 20 años, el 52\% culminó hasta el nivel medio (lo cual significa que cursó y culminó el grado 11), el $37 \%$ ya culminó un nivel técnico y el $11 \%$ restante un nivel tecnológico.

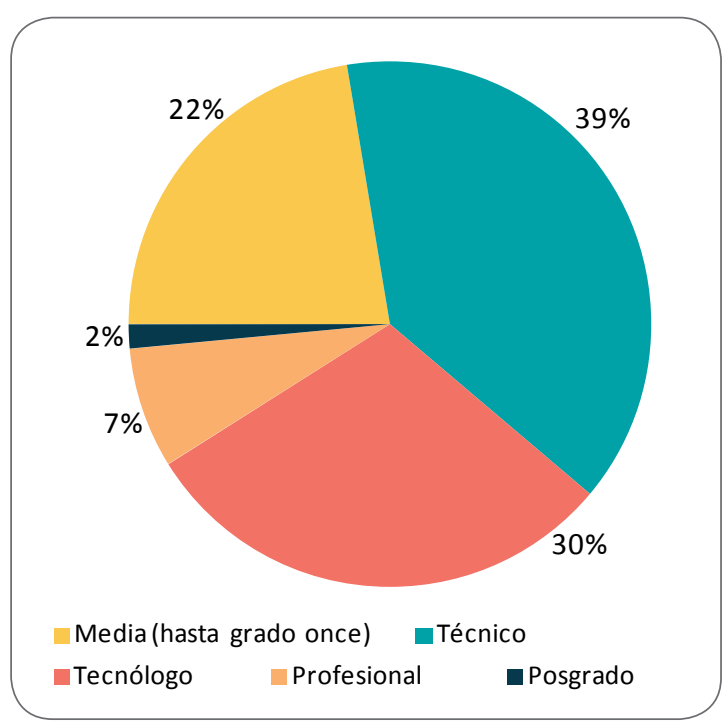

Figura 27. Nivel educativo culminado actualmente. Elaboración propia.
El 39\% de las mujeres de este grupo tiene un nivel técnico, el $30 \%$ un nivel tecnológico, el $22 \%$ un nivel medio (hasta grado 11), el 7\% un nivel profesional y el $2 \%$ restante completó un posgrado.

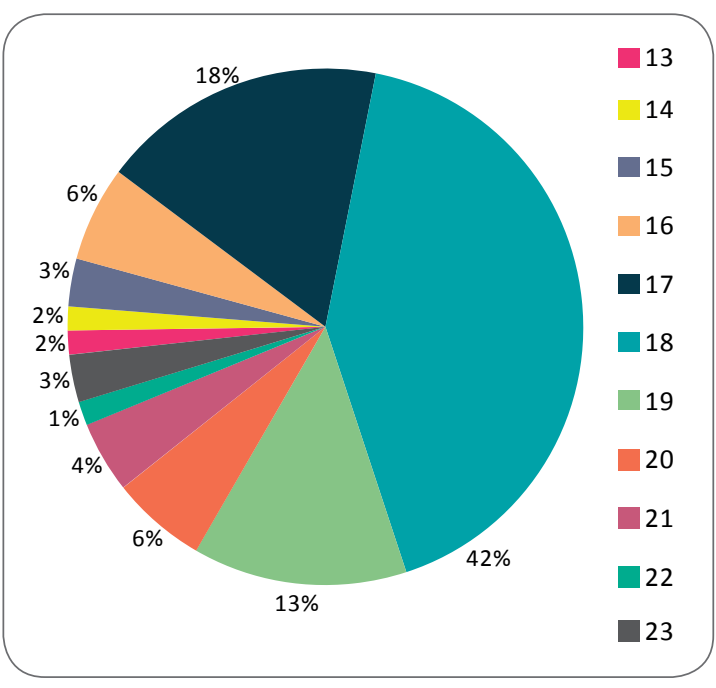

Figura 28. Edad en la que ingresó a su vida laboral: Elaboración propia.

Con relación a la edad en la que iniciaron su vida laboral, el $42 \%$ lo hizo cuando tenía 18 años de edad, el $7 \%$ en el rango de los 13 y 15 años, el $24 \%$ entre los 16 y 17 años, el 23\% ingresó entre los 19 y 21 , y el $4 \%$ restante ingresó entre los 22 y los 23 años de edad.

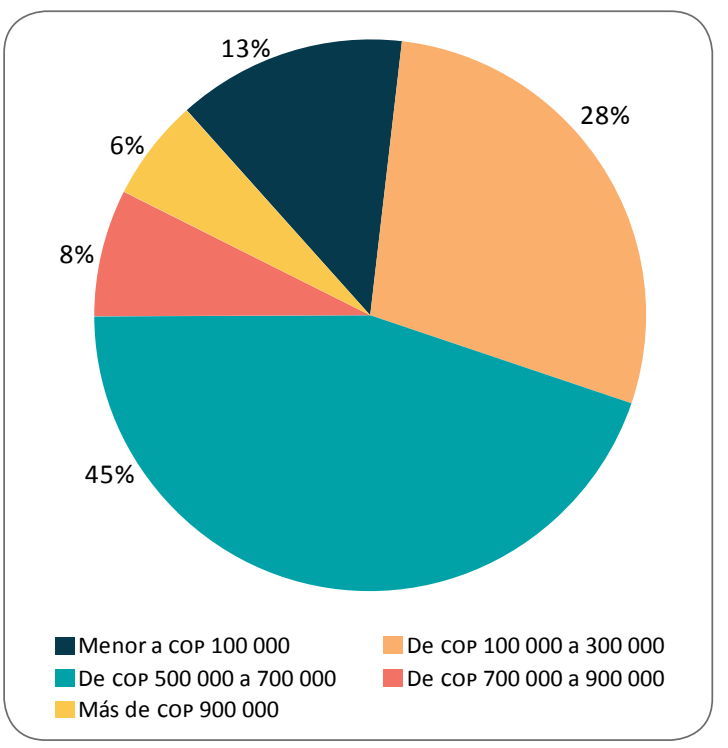

Figura 29. Promedio de ingreso antes de cumplir 20 años. Elaboración propia. 
Con respecto al promedio de ingresos que tenía este grupo de mujeres antes de cumplir 20 años, el 13\% tenía ingresos menores a COP 100 000, el 28\% entre los COP 100000 y los COP 300 000, el 45\% entre los cop 500000 y los cop 700000 , el 8\% entre los COP 700000 y los COP 900000 , y el 6\% restante tenía ingresos mayores a COP 900000 .

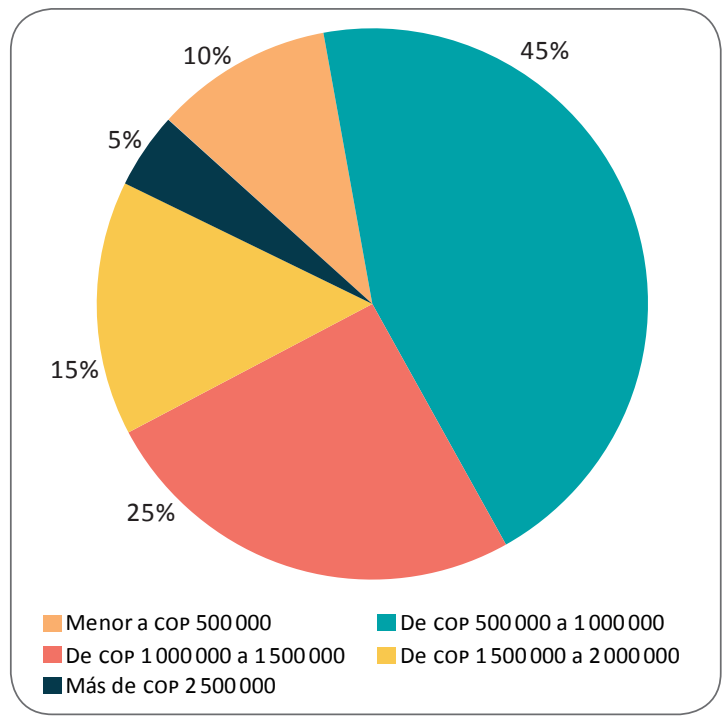

Figura 30. Promedio de ingreso actual. Elaboración propia.

En la actualidad, solo el 10\% cuenta con ingresos menores a COP 500 000, el $45 \%$ entre COP 500000 y cop 1000 000, el 25\% entre 1000000 y COP 1500 000, el 15\% entre COP 1500000 y COP 2000 000, y el 5\% restante de más de cop 2500000 .

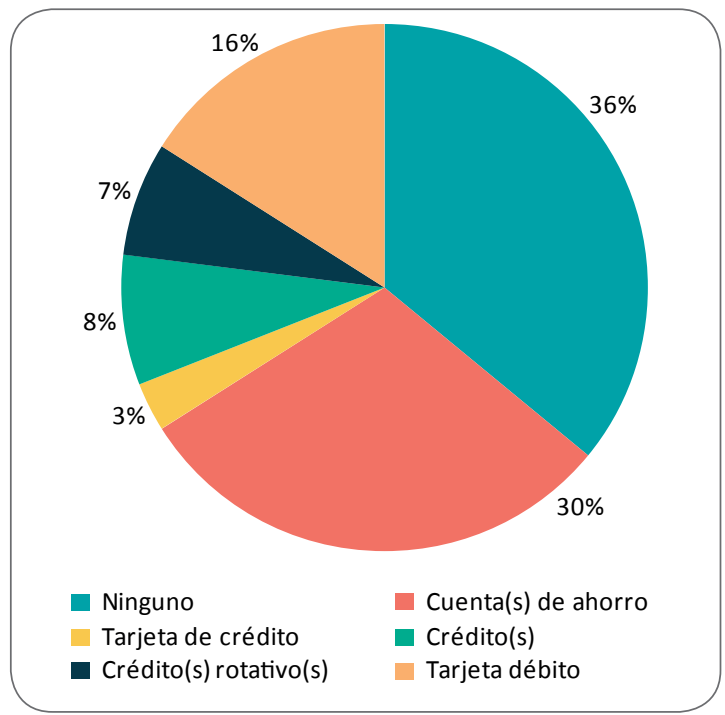

Figura 31. Productos del sistema financiero antes de cumplir 20 años. Elaboración propia.
Para antes de los 20 años, el 36\% no tenía ningún producto financiero, el $30 \%$ contaba con una cuenta de ahorros, el 3\% con una cuenta corriente, el $8 \%$ con una tarjeta de crédito, el $7 \%$ con un crédito y el $16 \%$ con tarjeta débito.

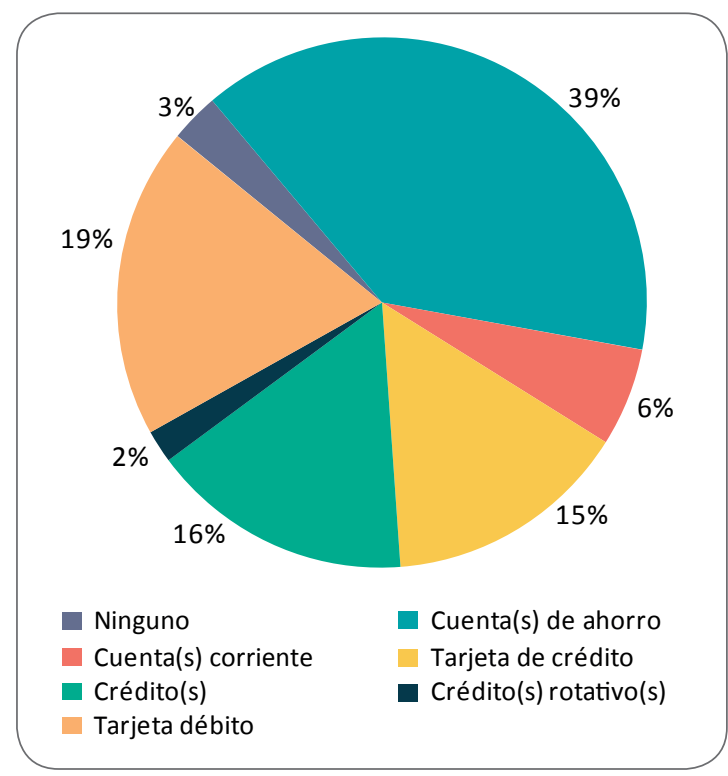

Figura 32. Productos del sistema financiero con los que actualmente cuenta. Elaboración propia.

En la actualidad, el 39\% cuenta con una cuenta de ahorros, el 6\% con una cuenta corriente, el 15\% con una tarjeta de crédito, el $16 \%$ con un crédito, el $2 \%$ tiene un crédito rotativo, el $19 \%$ con una tarjeta débito, y solo el $3 \%$ no cuenta con algún producto financiero.

\section{Determinantes del embarazo en adolescentes}

En esta sección se plantea el modelo Logit binomial, el cual se basa en la formulación de las variables independientes y la dependiente que pueden usarse para la aplicación de un modelo de regresión, lo que incluye distintas categorías que, en este caso, son de impacto o contexto social, de las cuales se puedan obtener o encontrar sus impactos y las relaciones que entre las variables se pueden dar de manera estadística. De esta manera, se llega a que la estimación y las conclusiones de este modelo sean más confiables y se acompañen de rigurosidad estadística a fin de comprobar su veracidad. En este modelo se utiliza una variable endógena 
$\left(y_{i}\right)$ discreta, a fin de analizar los determinantes del embarazo en adolescentes (mujeres menores de 20 años) que hayan sido madres o no lo hayan sido, con el fin de predecir la probabilidad $(\rho)$ de que dicho evento ocurra o no, dado un conjunto de regresores $\left(X_{i}\right)$.

Una interpretación estructural del modelo bajo el enfoque de la teoría de la utilidad (aleatoria) derivada de la selección, muestra que la población encuestada se enfrentó a la decisión de ser madre adolescente o no serlo $\left(y_{i}=0\right)$ o no $\left(y_{i}=1\right)$.

Según (Misas, 2011), las características socioeconómicas de cada grupo y de la atracción que ejerzan las alternativas dependerá la probabilidad de elección, así:

- $U_{i 0}=$ utilidad para el estudiante i cuando se es madre adolescente

- $U_{i 1}=$ utilidad para el estudiante i cuando no se es madre adolescente

- $\bar{U}_{i 0}=$ utilidad promedio para el estudiante $\mathrm{i}$ cuando se es madre adolescente

- $\bar{U}_{i 1}=$ utilidad promedio para el estudiante $\mathrm{i}$ cuando no se es madre adolescente

- $e_{i 0}=$ perturbaciones aleatorias para el estudiante i cuando se es madre adolescente

$\rho_{i}=\operatorname{prob}\left(y_{i}=1\right)=\operatorname{prob}\left(U_{i 1}>U_{i 0}\right)=$

$\operatorname{prob}\left(y_{i}^{*}>0\right)=\operatorname{prob}\left(e_{i}^{*}>X_{i}^{\prime} \beta\right)$

$\rho_{i}=1-\operatorname{prob}\left(e_{i}^{*} \leq-X_{i}^{\prime} \beta\right)=1-F\left(-X_{i}^{\prime} \beta\right)=F\left(X_{i}^{\prime} \beta\right)$

- $e_{i 1}=$ perturbaciones aleatorias para el estudiante i cuando no se es madre adolescente.

- $z_{i 0}^{\prime}=$ características de ser madre adolescente por el estudiante $i$.

- $z_{i 1}^{\prime}=$ características de no ser madre adolescente por el estudiante $i$.

- $w_{i}=$ características socioeconómicas de estudiante $\mathrm{i}$.

La alternativa seleccionada será aquella que maximice la utilidad esperada, dado que la estudiante no tendrá embarazo adolescente si y solo si considera que la ganancia esperada de no tenerlo es mayor que si lo tiene. Se toma una variable latente:

$$
y_{i}^{*}=U_{i 1}-U_{i 0}
$$

$$
y_{i}=\left\{\begin{array}{llll}
1 & \text { si } & y_{i}^{*}>0 & U_{i 1}>U_{i 0} \\
0 & \text { si } & y_{i}^{*} \leq 0 & U_{i 1}<U_{i 0}
\end{array}\right\}
$$

$$
y_{i}^{*}=\left(z_{i 1}-z_{i 0}\right)^{\prime} \delta+w_{i}^{\prime}\left(y_{1}-y_{0}\right)+\left(e_{i 1}-e_{i 0}\right)
$$

$$
y_{i}^{*}=\left[\left(z_{i 1}-z_{i 0}\right)^{\prime}, w_{i}^{\prime}\right]\left[\begin{array}{c}
\delta \\
y_{1}-y_{0}
\end{array}\right]+e_{i}^{*}
$$

$$
y_{i}^{*}=X^{\prime} \beta+e_{i}^{*}
$$

La estudiante decidirá asumir no tener embarazos en su adolescencia cuando:

- La función de distribución de la perturbación aleatoria $e_{i}^{*}$ definirá el tipo de modelo que se especificará, $\mathrm{F}(\mathrm{x})$ es una función de probabilidad acumulada que garantiza un rango entre cero $y$ uno. Si se distribuye uniformemente es un modelo lineal de probabilidad:

$\left.[\mathrm{P}(\mathrm{Y})]_{i}=1\right)=\mathrm{F}(\mathrm{X} \beta)$

- Cuando la función de distribución acumulada es logística, se estima un modelo Logit:

$\left.[\mathrm{P}(\mathrm{Y})]_{i}=1 \backslash X_{i}\right)=\frac{e^{X \beta}}{\left(1+e^{X \beta}\right)}$

- Cuando la función de distribución acumulada es una normal estándar, se estima un modelo Probit:

$\left.[\mathrm{P}(\mathrm{Y})]_{i}=1 \backslash X_{i}\right)=\int_{-\infty}^{X \beta} \emptyset(z) d z=\frac{1}{\sqrt{2 \pi}} e^{-X \beta^{z} / 2}$ 


\section{Descripción de variables}

A continuación, se presentan la media, la desviación estándar, el mínimo y el máximo de cada una de las variables utilizadas en las regresiones lineales propuestas.

Tabla 1

Variables en regresiones lineales

\begin{tabular}{|r|rrrrr|}
\hline . summarize embado ingreso nivedu grufam prodfin & \\
Variable & Obs & Mean & Std. Dev. & Min & Max \\
\hline embado & 201 & .3333333 & .4725816 & 0 & 1 \\
ingreso & 201 & 1.507463 & 1.487008 & 0 & 5 \\
nivedu & 201 & 2.169154 & .8253749 & 0 & 4 \\
grufam & 201 & 4.268657 & 1.648473 & 1 & 10 \\
prodfin & 201 & .2537313 & .4362322 & 0 & 1 \\
\hline
\end{tabular}

Nota. Salidas del software Stata versión 10. Elaboración propia.

El modelo conceptual que se propone a fin de relacionar la probabilidad de que una mujer sea madre adolescente y sus determinantes se presenta a continuación:

embado $=\beta_{1}$ ingreso $+\beta_{2}$ nivedu + $\beta_{3}$ grufam $+\beta_{4}$ prodfin

\section{Donde,}

- Embarazo en adolescencia (embado). Esta es una variable dicotómica que se construyó al tomar dos posibles valores, 0 y 1 , dependiendo si fue madre en la adolescencia o no lo fue, tal como se presenta en la tabla 2.

Tabla 2

Embarazo en adolescencia

\begin{tabular}{ll}
\hline Madres en la adolescencia & 0 \\
Número de madres en la adolescencia & 1 \\
\hline
\end{tabular}

Nota. Elaboración propia.

- Ingreso (ingreso). Es una variable que se construyó de acuerdo con las respuestas seleccionadas durante la adolescencia, como se puede observar en la tabla 3.
Tabla 3

Nivel de ingreso

\begin{tabular}{cc}
\hline$<$ a 100000 & 0 \\
De 100001 a 300000 & 1 \\
De 300001 a 500000 & 2 \\
De 500001 a 700000 & 3 \\
De 700001 a 900000 & 4 \\
$>900000$ & 5 \\
\hline
\end{tabular}

Nota. Elaboración propia.

- Nivel educativo (nivedu). La variable se construyó mediante la asignación de valores enteros a cada nivel terminado por las encuestadas en la adolescencia, de la manera que se presenta en la tabla 4.

Tabla 4

Nivel educativo

\begin{tabular}{ll} 
Primaria & 0 \\
Hasta noveno grado & 1 \\
Bachiller & 2 \\
Técnico & 3 \\
Tecnólogo & 4 \\
\hline
\end{tabular}

Nota. Elaboración propia.

- Composición del grupo familiar (grufam). Es una variable que se construyó de acuerdo con el número de integrantes del grupo familiar de las mujeres encuestadas durante su adolescencia.

- Productos financieros (prodfin). Es una variable dicotómica que se construyó al tomar valores de 0 y 1 ; en el caso de no haber tenido productos financieros en su adolescencia se le asigna el valor de 0 , y en el caso de haberlos tenido se le asigna 1 .

\section{Hipótesis de los determinantes del embarazo en adolescentes}

- $h_{1}=$ Se espera una relación positiva, es decir, a mayor ingreso en la adolescencia de las encuestadas mayor probabilidad de no haber sido madre adolescente. 
- $h_{2}=$ Se espera una relación positiva, es decir, a mayor nivel educativo en la adolescencia de las encuestadas mayor probabilidad de no haber sido madre adolescente.

- $h_{3}=$ Se espera una relación positiva, es decir, a mayor cantidad de integrantes del grupo familiar en la adolescencia de las encuestadas mayor probabilidad de no haber sido madre adolescente.

- $h_{4}=$ Se espera una relación positiva, es decir, a mayor cantidad de productos financieros en la adolescencia, mayor probabilidad de no haber sido madre adolescente.

De la anterior salida del modelo Logit Binomial, de la cual se toma como criterio de validación el R2 - el cual indica cómo las variables independientes ingreso, nivedu, grufam y prdodfin explican el comportamiento de la variable dependiente embado en un 20,15\% - además, se tomó un intervalo de confianza del 95\%, del cual se deriva el análisis de la significancia conjunta de los parámetros o variables independientes, y cómo estás explican la variable dependiente.

\section{H0: Todos los coeficientes estimados son estadísticamente iguales a cero}

Para tal fin, se realizó la prueba del test de Wald, la cual sigue una distribución Chi-cuadrado y evalúa la significancia global del modelo.

El resultado de este estadístico es Prob > 0,0000 , es decir, es un valor inferior a un nivel de significancia $(\alpha=0,05)$. Por tanto, el embarazo en adolescentes se explica por las variables independientes en conjunto con una probabilidad de acierto mayor al 95\%, y se puede rechazar la hipótesis nula por la cual se establece que todos los coeficientes estimados son iguales a cero, de manera que existe un modelo y este es capaz de explicar la variable dependiente con un índice de confianza alto.

$\mathrm{Al}$ continuar con el análisis de la salida se observa el estadístico $\mathrm{Z}$ que sigue una distribución normal estándar, con el cual se comprueba la significancia individual de cada uno de los coeficientes del modelo cuando la probabilidad es inferior al nivel de significancia $(a=0,05)$, es decir, cada variable independiente cuya prueba $\mathrm{Z}$ sea menor o

Tabla 5

Determinantes del embarazo en adolescentes

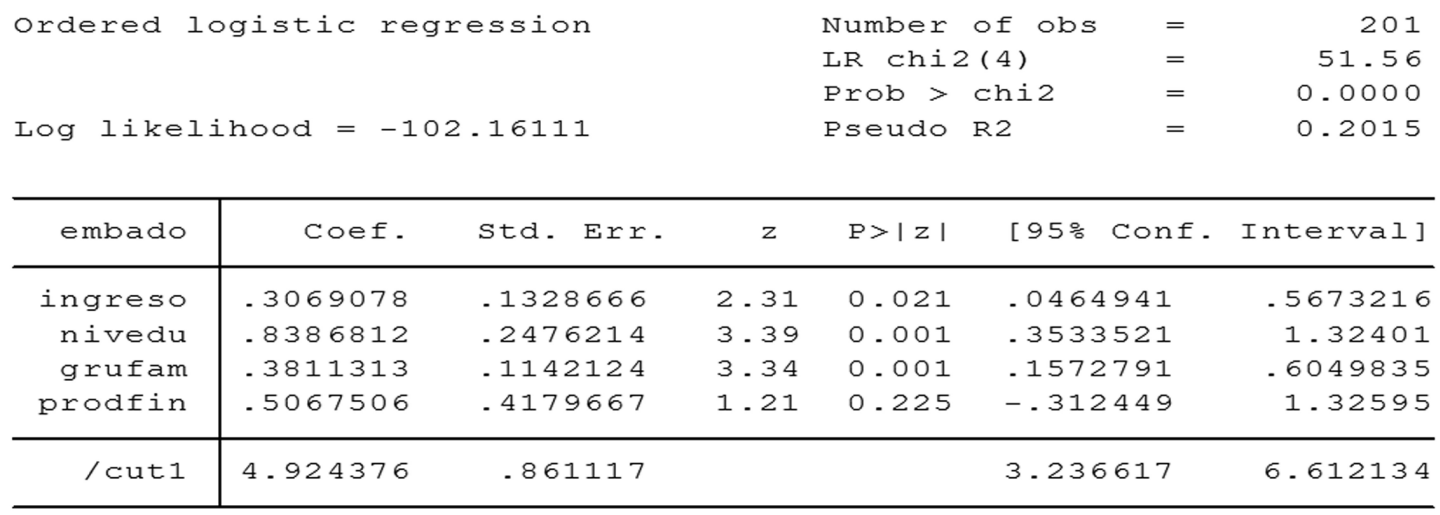

\footnotetext{
- ologit embado ingreso nivedu grufam prodxin

Iteration 0 :

Iteration 1 :

Iteration 2 :

Iteration 3 :

Iteration 4 :
} 
igual a 0,05 es una variable significativa y explica el embarazo en adolescentes de forma independiente, con excepción de los productos financieros.

También se analiza la bondad de ajuste del modelo que corresponde al valor del $\mathrm{R} 2$ y en este caso de investigación es de 0,2015, valor considerado para este tipo de modelos Logit de buena calidad de ajuste y que se interpreta como el porcentaje que las variables independientes explican a la variable dependiente, en este caso el embarazo en adolescentes. Además, se confirma el valor de la prueba conjunta del test de Wald, y se valida así la importancia del modelo en general.

A partir del planteamiento según el cual la importancia de un modelo Logit radica en los signos, se procede a verificar las hipótesis planteadas de las variables que permanecen en el modelo, en el que se observa que todas las hipótesis se cumplen al tener signos positivos entre las variables independientes y la variable dependiente. Además, son significativas al 95\% de confianza en el caso del ingreso, el nivel educativo y en la conformación del grupo familiar. Solo la tenencia de productos financieros no es significativa (el cálculo de los efectos marginales se presenta en la tabla 6).

Los efectos marginales se calculan tanto para el evento de ser madre adolescente como de no serlo.

Según los efectos marginales, la probabilidad de ser madre en la adolescencia para la población encuestada es del $71 \%$, de manera que los factores más relevantes para esto son el nivel educativo, la conformación del grupo familiar y los ingresos durante esta etapa de su vida.

Respecto a las variaciones en los regresores o en las variables explicativas, se encuentra que al pasar una adolescente a mejorar su nivel de ingreso en un nivel - de acuerdo con la escala propuesta en esta investigación-, su probabilidad de ser madre adolescente se reduce en un $6,34 \%$, así como su probabilidad de no ser madre adolescente aumenta en un $6,34 \%$.

Tabla 6

Efectos marginales

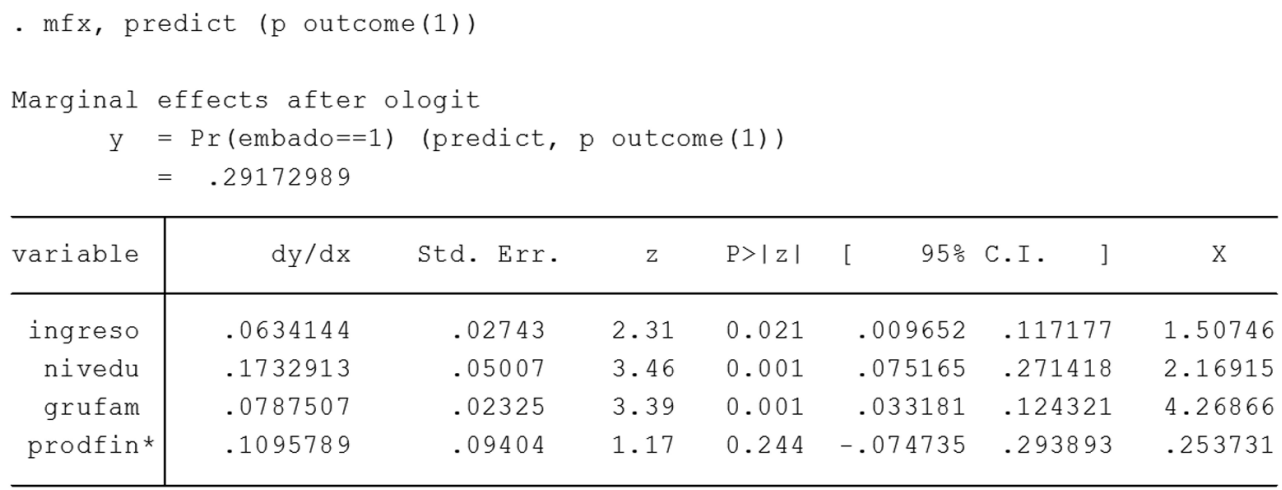

(*) dy/dx is for discrete change of dummy variable from 0 to 1

- mfx, predict (p outcome (0))

Marginal effects after ologit

$\mathrm{y}=\operatorname{Pr}($ embado $==0) \quad($ predict, $\mathrm{p}$ outcome $(0))$

$=.70827011$

\begin{tabular}{|c|c|c|c|c|c|c|c|}
\hline variable & $d y / d x$ & std. Err. & $z$ & $P>|z|$ & $95 \%$ & C.I. & $\mathrm{x}$ \\
\hline ingreso & -.0634144 & .02743 & -2.31 & 0.021 & -.117177 & -.009652 & 1.50746 \\
\hline nivedu & -.1732913 & .05007 & -3.46 & 0.001 & -.271418 & -.075165 & 2.16915 \\
\hline grufam & -.0787507 & .02325 & -3.39 & 0.001 & -.124321 & -.033181 & 4.26866 \\
\hline prodfin* & -.1095789 & .09404 & -1.17 & 0.244 & -.293893 & .074735 & .253731 \\
\hline
\end{tabular}

(*) dy/dx is for discrete change of dummy variable from 0 to 1

Nota. Elaboración propia. 
Cuando una mujer en la adolescencia avanza en un grado escolar su probabilidad de ser madre adolescente se reduce en $17,32 \%$ o cuando se reduce su nivel educativo bien sea por deserción u otro factor su probabilidad de ser madre adolescente aumenta $17,32 \%$. Cuando una mujer hace parte de un grupo familiar más numeroso, por cada integrante adicional su probabilidad de ser madre adolescente disminuye en un 7,87\%, o viceversa. Finalmente, para el acceso a productos financieros está probabilidad no es significativa.

\section{Impacto del embarazo en adolescentes en los ejes fundamentales de nivel educativo e ingreso}

Finalmente, esta sección pretende medir el impacto de los embarazos en adolescentes tanto en el nivel educativo como en el nivel de ingresos. A fin de medir cada uno de estos impactos se tomaron datos actuales de las mujeres encuestadas que en la actualidad cuentan con un nivel de ingresos y un nivel educativo determinado, para lo cual cada uno de estos ejes fundamentales se toma como variable dependiente $y$, dentro de las variables independientes, se incluye el haber sido o no madre en la adolescencia, lo que permite visibilizar su impacto en estos aspectos. Para esto se llevaron a cabo dos regresiones lineales, de la siguiente manera:

$\widehat{Y}=\beta_{1} x+\beta_{2} z+\beta_{3} I$

Donde,

$\hat{Y}=$ variable dependiente (nivel educativo o nivel de ingresos, según sea el caso).

$X$ : variable independiente 1 , según sea el caso. $Z$ : variable independiente 2 , según sea el caso. I: variable independiente 3 , según sea el caso.

\section{Especificación del modelo}

Se parte de la minimización del error por el método de mínimos cuadrados ordinarios, con el objetivo de encontrar los parámetros de la ecuación de regresión lineal $(\beta 1, \beta 2, \beta 3)$, de modo que se llegue al sistema de tres ecuaciones tres incógnitas, el cual se puede resolver por sustitución, Cramer, eliminación, etc., lo que da como resultado los mejores parámetros que explican el comportamiento de la variable dependiente a partir de las variables independientes, como se muestra a continuación.

$$
\begin{gathered}
\varepsilon^{2}=\sum_{i=1}^{n}(Y-\hat{Y}) \\
\varepsilon^{2}=\sum_{i=1}^{n}\left(Y-\beta_{1} x-\beta_{2} z-\beta_{3} I\right)^{2} \\
\frac{d \varepsilon^{2}}{d \beta_{1}}=2 \sum_{i=1}^{n}\left(Y-\beta_{1} x-\beta_{2} z-\beta_{3} I\right)(-x) \\
0-2 \sum_{i=1}^{n} Y x+2 \beta_{1} \sum_{i=1}^{n} x^{2}+2 \beta_{2} \sum_{i=1}^{n} z x+2 \beta_{3} \sum_{i=1}^{n} I x \\
2 \sum_{i=1}^{n} Y x=2 \beta_{1} \sum_{i=1}^{n} x^{2}+2 \beta_{2} \sum_{i=1}^{n} z x+2 \beta_{3} \sum_{i=1}^{n} I x \\
\sum_{i=1}^{n} Y x=\beta_{1} \sum_{i=1}^{n} x^{2}+\beta_{2} \sum_{i=1}^{n} z x+\beta_{3} \sum_{i=1}^{n} I x \\
\frac{d \varepsilon^{2}}{d \beta_{2}}=2 \sum_{i=1}^{n}\left(Y-\beta_{1} x-\beta_{2} z-\beta_{3} I\right)(-z) \\
2 \sum_{i=1}^{n} Y z=2 \beta_{1} \sum_{i=1}^{n} x z+2 \beta_{2} \sum_{i=1}^{n} z^{2}+2 \beta_{3} \sum_{i=1}^{n} I z
\end{gathered}
$$$$
\sum_{i=1}^{n} Y Z=\beta_{1} \sum_{i=1}^{n} x z+\beta_{2} \sum_{i=1}^{n} z^{2}+\beta_{3} \sum_{i=1}^{n} I Z
$$

$$
\begin{gathered}
\frac{d \varepsilon^{2}}{d \beta_{3}}=2 \sum_{i=1}^{n}\left(Y-\beta_{1} x-\beta_{2} Z-\beta_{3} I\right)(-I) \\
0=-2 \sum_{i=1}^{n} Y I+2 \beta_{1} \sum_{i=1}^{n} x I+2 \beta_{2} \sum_{i=1}^{n} z I+2 \beta_{3} \sum_{i=1}^{n} I^{2} \\
2 \sum_{i=1}^{n} Y I=2 \beta_{1} \sum_{i=1}^{n} x I+2 \beta_{2} \sum_{i=1}^{n} z I+2 \beta_{3} \sum_{i=1}^{n} I^{2}
\end{gathered}
$$$$
\sum_{i=1}^{n} Y I=\beta_{1} \sum_{i=1}^{n} x I+\beta_{2} \sum_{i=1}^{n} z I+\beta_{3} \sum_{i=1}^{n} I^{2}
$$ 


\section{Descripción de variables}

A continuación, se presentan la media, la desviación estándar, el mínimo y el máximo de cada una de las variables utilizadas en las regresiones lineales propuestas.

Tabla 7

Regresiones lineales

. summarize embado edad nivedu ingreso inilab

\begin{tabular}{r|rrrrr} 
Variable & Obs & Mean & Std. Dev. & Min & Max \\
\hline embado & 201 & .3333333 & .4725816 & 0 & 1 \\
edad & 201 & 27.70149 & 5.121567 & 21 & 40 \\
nivedu & 201 & 1.20398 & .8678618 & 0 & 4 \\
ingreso & 201 & 1.81592 & 1.127362 & 0 & 5 \\
inilab & 201 & 17.87562 & 1.868008 & 12 & 24
\end{tabular}

Nota. Elaboración propia.

- Embarazo en adolescencia (embado). Esta es una variable dicotómica que se construyó al tomar dos posibles valores, 0 y 1 , dependiendo de si fue madre en la adolescencia o no lo fue, tal como se muestra en la tabla 8.

Tabla 8

Embarazo en adolescencia

\begin{tabular}{ll}
\hline Madres en la adolescencia & 0 \\
No madres en la adolescencia & 1 \\
\hline
\end{tabular}

Nota. Elaboración propia.

- Edad (edad). Los valores de la edad corresponden a la edad actual de cada una de las mujeres encuestadas; la de menor edad fue de 21 años y la de mayor fue de 40 años, y la media fue 27,7 años.

- Nivel educativo culminado (nivedu). La variable se construyó mediante la asignación de valores enteros a cada nivel terminado por las encuestadas, tal como se muestra en la tabla 9.
Tabla 9

Nivel educativo

\begin{tabular}{ll}
\hline Media (hasta grado once) & 0 \\
Técnico & 1 \\
Tecnólogo & 2 \\
Profesional & 3 \\
Posgrado & 4 \\
\hline
\end{tabular}

Nota. Elaboración propia.

Tabla 10

Nivel de ingreso

\begin{tabular}{cc}
\hline$<$ a 500000 & 0 \\
De 500001 a 1.000000 & 1 \\
De 1000001 a 1500000 & 2 \\
De 1500001 a 2000000 & 3 \\
De 2000001 a 2500000 & 4 \\
$>2500000$ & 5 \\
\hline
\end{tabular}

Nota. Elaboración propia.

- Ingreso (ingreso). Es una variable que se construyó de acuerdo con las respuestas seleccionadas, tal como se presenta en la tabla 10.

- Valores de inicio de vida laboral (inilab). Corresponden a la edad en la cual las encuestadas iniciaron su vida laboral; la de menor edad fue de 12 años y la de mayor fue de 24 años (la media fue 17,8 años).

\section{Hipótesis para impacto en el nivel educativo}

- $h_{1}=$ se espera una relación positiva, es decir, a mayor edad actual de las encuestadas mayor nivel educativo se tendrá.

- $h_{2}=$ se espera una relación positiva, es decir, cuando las mujeres no son madres en la adolescencia, aumenta su nivel educativo en la actualidad.

- $h_{3}=$ se espera que a mayor ingreso del grupo de mujeres encuestadas exista un mayor nivel educativo. 
De esta manera, el modelo propuesto para explicar el nivel educativo es el siguiente:

$$
\begin{aligned}
& \text { nivedu }=\beta_{1} \text { edad }+\beta_{2} \text { embado } \\
& +\beta_{3} \text { ingreso }
\end{aligned}
$$

Para la salida anterior se toma como criterio de validación el R2, el cual indica que las variables independientes edad, embado e ingreso explican el comportamiento de la variable dependiente nivedu en un $67,65 \%$. Además, se tomó un intervalo de confianza del 95\%, el cual sirve como parámetro para las pruebas $\mathrm{T}$ y $\mathrm{F}$.

Respecto a la prueba $\mathrm{T}$ de cada variable independiente, se encontró que cada una es significativa individualmente para explicar el nivel educativo en la actualidad de las mujeres encuestadas al 95\% de confianza. Otro de los criterios utilizados para el modelo propuesto es la prueba $\mathrm{F}$, la cual mide la significancia del modelo en su conjunto y, en este caso, es significativo al 95\% de confianza.

Del mismo modo, las hipótesis propuestas fueron acordes al resultado y se encontró relación positiva en cada caso.

La ecuación que lo explica es:

nivedu $=0,0274$ edad $+0,2654$ embado $+0,1845$ ingreso

En esta se puede explicar que el embarazo en adolescentes afecta negativamente el nivel educativo de las mujeres, es decir, de acuerdo con los resultados obtenidos, cuando una mujer no tiene embarazos en la adolescencia su probabilidad de aumentar su nivel educativo se incrementa en un $26,54 \%$.

\section{Hipótesis para impacto en el ingreso}

- $h_{1}=$ se espera una relación positiva, es decir, a mayor edad actual de las encuestadas mayores ingresos tendrán.

- $h_{2}=$ se espera una relación positiva, es decir, cuando las mujeres no son madres en la adolescencia aumentan sus ingresos.

- $h_{3}=$ se espera que a mayor nivel educativo del grupo de mujeres encuestadas exista un mayor nivel de ingresos.

Para la salida anterior se toma como criterio de validación el R2, el cual indica que las variables independientes edad, embado y nivedu explican el comportamiento de la variable dependiente ingreso en un $77,30 \%$. Además, se tomó un intervalo de confianza del 95\%, el cual sirve como parámetro para las pruebas $\mathrm{T}$ y $\mathrm{F}$.

Respecto a la prueba $\mathrm{T}$ de cada variable independiente, se encontró que edad y nivedu son significativas individualmente para explicar los ingresos en la actualidad de las mujeres encuestadas al 95\% de confianza, mientras que la variable de interés embado no fue significativa. Otro de los criterios utilizados para el modelo propuesto es la prueba F, la cual mide la significancia del modelo en su

Tabla 11

\begin{tabular}{|c|c|c|c|c|c|c|c|}
\hline source & ss & df & \multicolumn{2}{|c|}{ MS } & & \multirow{2}{*}{$\begin{array}{l}\text { Number of obs } \\
\text { F( 3, 198) } \\
\text { Prob }>\text { F }\end{array}$} & \multirow{2}{*}{$\begin{array}{r}201 \\
138.05 \\
0.0000\end{array}$} \\
\hline Model & 299.033314 & 3 & \multicolumn{2}{|c|}{99.6777713} & & & \\
\hline Residual & 142.966686 & 198 & \multicolumn{2}{|c|}{.722053971} & & $\mathrm{R}$-squared & 0.6765 \\
\hline Total & 442 & 201 & 2.1 & 0498 & & $\begin{array}{l}\text { Adj R-squared } \\
\text { Root MSE }\end{array}$ & .84974 \\
\hline nivedu & Coef. & std. & Err. & $t$ & $P>|t|$ & [95\% Conf. & Interval] \\
\hline edad & .0274782 & .0045 & 923 & 5.98 & 0.000 & .0184221 & .0365342 \\
\hline embado & .2654878 & .1220 & 237 & 2.18 & 0.031 & .024855 & .5061207 \\
\hline ingreso & .1845388 & .05 & 542 & 3.21 & 0.002 & .0713056 & .297772 \\
\hline
\end{tabular}

Impacto del embarazo en adolescentes en el nivel educativo 
Tabla 12

Impacto del embarazo en adolescentes en el ingreso

\begin{tabular}{|c|c|c|c|c|c|c|c|}
\hline Source & ss & $d f$ & & MS & & \multirow{3}{*}{$\begin{array}{l}\text { Number of obs } \\
\text { F( 3, 198) } \\
\text { Prob > F }\end{array}$} & \multirow{2}{*}{$\begin{array}{r}201 \\
224.77\end{array}$} \\
\hline & & & \multirow{2}{*}{\multicolumn{2}{|c|}{236.286026}} & & & \\
\hline Model & 708.858078 & 3 & & & & & 0.0000 \\
\hline Residual & 208.141922 & 198 & \multicolumn{2}{|c|}{1.05122183} & & R-squared & 0.7730 \\
\hline Total & 917 & 201 & 4.5 & 18905 & & $\begin{array}{l}\text { Adj R-squared } \\
\text { Root MSE }\end{array}$ & $=1.0253$ \\
\hline ingreso & Coef. & std. & Err. & $\mathrm{t}$ & $P>|t|$ & [95\& Conf. & Interval] \\
\hline edad & .0563915 & .0044 & 938 & 12.55 & 0.000 & .0475297 & .0652534 \\
\hline embado & -.1565917 & .1485 & 669 & -1.05 & 0.293 & -.4495681 & .1363847 \\
\hline nivedu & .2686658 & .0835 & 964 & 3.21 & 0.002 & .1038122 & .4335194 \\
\hline
\end{tabular}

Nota. Elaboración propia.

conjunto y, en este caso, es significativo al 95\% de confianza.

Del mismo modo, dos de las hipótesis propuestas fueron acordes al resultado, de modo que se encontró relación positiva en cada caso. Sin embargo, en el caso de embado el signo es negativo y no coincide con la hipótesis propuesta.

La ecuación que lo explica es:

ingreso $=0,05639$ edad $-0,15659$ embado

$+0,26866$ nivedu

En esta se puede explicar que el embarazo en adolescentes afecta positivamente el nivel de ingresos de las mujeres, es decir, de acuerdo con los resultados obtenidos, cuando una mujer no tiene embarazos en la adolescencia su probabilidad de aumentar su nivel de ingresos disminuye en un $15,65 \%$. Esto no es acorde con lo propuesto en esta investigación, pero puede acreditarse al hecho de que la población encuestada es una población estudiantil que, en el caso de las mujeres más jóvenes, no tiene ingresos por el hecho de no estar trabajando, y a su vez son quienes componen en su mayoría el grupo de mujeres que no fueron madres en la adolescencia.

\section{Conclusiones}

Algunas características sociales y económicas de una población determinada influyen notoriamente en la posibilidad de tener embarazos adolescentes en las mujeres menores de 20 años, es decir, el entorno de las mujeres se ve afectado por estas condiciones.

Dentro de los resultados de la investigación y las conclusiones a las que se llegó, se apoyan las deducciones a las que llegó el DANE y el Ministerio de Educación, en el sentido de que tratan sobre el impacto económico que se produce cuando se queda en embarazo en la adolescencia, bien sea mediante la deserción o bien mediante la desaceleración del aumento del nivel educativo. Sin embargo, no se pueden comparar resultados de manera específica, pues no se encontró una investigación semejante o paralela a esta.

Particularmente, tanto el nivel de ingresos como el nivel educativo que rodea a una adolescente se relacionan positivamente con la probabilidad de no tener embarazos en la adolescencia, es decir, a mayores niveles de ingreso, mayor la probabilidad de no ser madre adolescente. Lo mismo ocurre con el nivel educativo: a mayor nivel educativo mayor la probabilidad de no ser madre adolescente. 
En el caso de la conformación del grupo familiar de la adolescente, la probabilidad de no tener embarazos adolescentes aumenta con el número de integrantes del núcleo familiar.

El acceso a productos financieros, por sí solo, no es significativo para explicar el embarazo en adolescentes, es decir, a nivel individual no hay una relación de largo plazo entre esta variable y el embarazo en adolescentes, aunque en teoría existe una relación positiva: mayor acceso a productos financieros aumenta la probabilidad de no tener embarazos adolescentes.

El modelo propuesto en conjunto es significativo, lo cual significa que todas las variables independientes relacionadas como un conjunto de características explican el embarazo en adolescentes.

Los modelos en los que la variable dependiente fueron el nivel de ingresos y el nivel educativo se explican por el fenómeno de haber tenido o no un embarazo en la adolescencia.

En particular, tener un embarazo en la adolescencia afecta negativamente el nivel educativo en el futuro, es decir, un embarazo a temprana edad causa retrasos en la edad que alcanzan los diferentes niveles educativos, a partir de la premisa de que la muestra utilizada en este estudio es población universitaria.

Se encontró que el embarazo en adolescentes hace que el nivel de ingresos a futuro aumente, algo que no se esperaba en la investigación. Sin embargo, esto es factible que se deba a la población con la que se trabajó, ya que esta es población universitaria y la mayor parte de las mujeres que fueron madres adolescentes tienen una vida laboral mayor a la de las mujeres que no fueron madres adolescentes. Lo anterior si se parte de la edad actual, es decir, las que no fueron madres adolescentes son menores en la actualidad con relación a las que sí lo fueron y tienen una vida laboral mucho menor, lo cual se refleja en los niveles de ingresos.

Se espera que el estudio realizado muestre no solo las causas, sino también las consecuencias de un embarazo en adolescentes, de modo tal que esto se pueda llevar a las políticas públicas que intervienen en este fenómeno, mediante las mediciones realizadas y los datos cuantitativos obtenidos.

\section{Referencias}

Davidson, R. y MacKinnon, J. (2004). Chapter 11: Discrete and limited dependent variables. En Econometric Theory and Methods (pp. 451-475).

Greene, W. (2007). Ordered data. En Econometric Analysis (pp. 736-740). New York University, Prentice Hall.

Mejía-Mendoza, M., Laureano-Eugenio, J., Gil-Hernández, E., Ortiz-Villalobos, R., Blackaller-Ayala, J. y Benítez-Morales, R. (2015, octubre-diciembre). Condiciones socioculturales y experiencia del embarazo en adolescentes de Jalisco, México: estudio cualitativo. Revista Colombiana de Obstetricia y Ginecología, 66(4).

Mendoza, W. y Subiría, G. (2013). El embarazo adolescente en el Perú: situación actual e implicancias para las políticas públicas. Revista Peruana de Medicina Experimental y Salud Pública, 30(3). Recuperado de: http://www.rpmesp.ins.gob.pe/ index.php/rpmesp/ article/view/286/2188

Organizacion Mundial de la Salud-oms. (2014, septiembre). El embarazo en la adolescencia. Recuperado de http://www.who.int/mediacentre/factsheets /fs364/es/

Parada, A. (2005, marzo 25). El embarazo adolescente le cuesta al país. UN Periódico, (72), 25.

Salazar-Arango, A., Acosta-Murcia, M. M., Lozano-Restrepo, N. y Quintero-Camacho, M. C. (2008, julio-diciembre). Consecuencias del embarazo adolescente en el estado civil de la madre joven: estudio piloto en Bogotá, Colombia. Persona y Bioética, 12(2), 169-182.

Salazar, A., Rodríguez, L. y Daza, R. (2009). Embarazo y maternidad adolescente en Bogotá y municipios aledaños consecuencias en el estudio, estado civil, estructura familiar, ocupación y proyecto de vida. Persona y Bioética, 11(2). Recuperado de http://personaybioetica.unisabana.edu.co/index.php/personaybioetica/article/view/951/1929

Welti, C. (2000). Análisis demográfico de la fecundidad adolescente en México. Papeles de población. Universidad Nacional Autónoma de México. Recuperado de: http://repositoriodigital.academica.mx/jspui/ handle/987654321/227893 